\title{
Trajectory Tracking Control of a Double-pendulum System
}

Jose Luis Briceño Pérez

(j1.briceno10@uniandes.edu.co)

Adviser: Carlos Francisco Rodríguez Herrera

(crodrigu@uniandes.edu.co)

Universidad de los Andes, Colombia 


\section{Content}

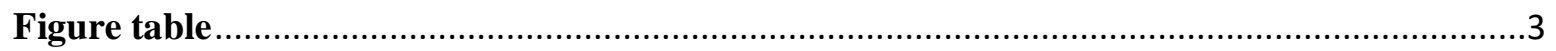

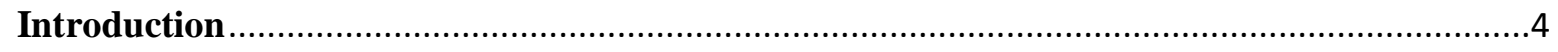

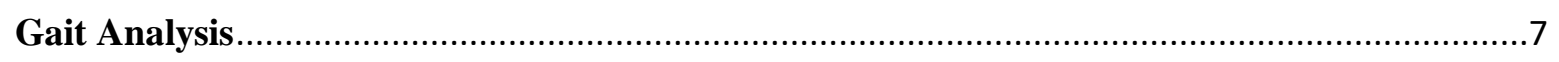

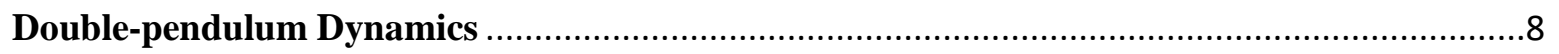

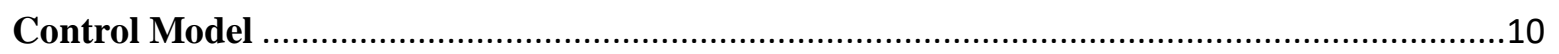

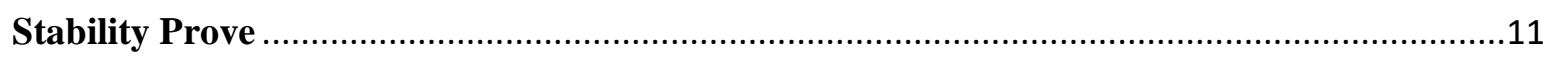

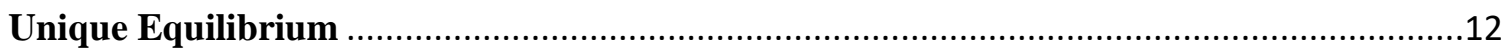

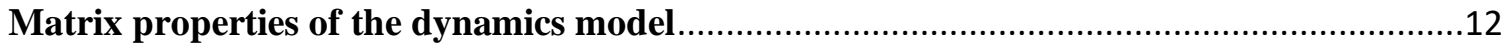

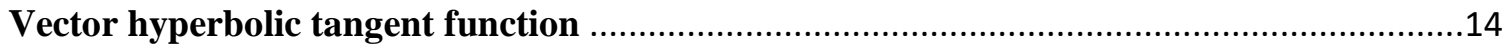

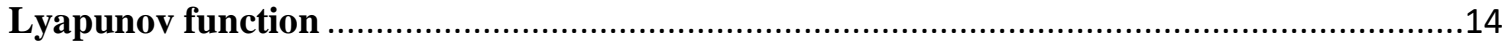

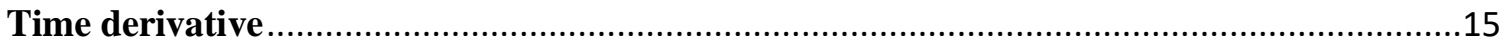

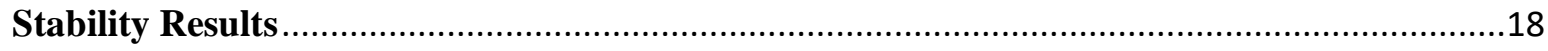

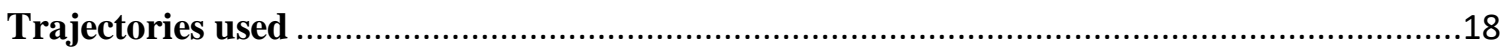

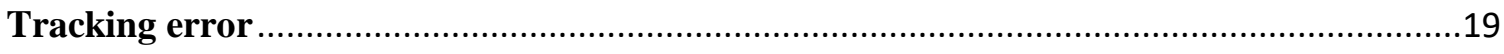

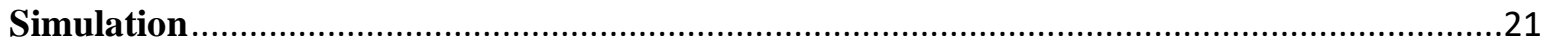

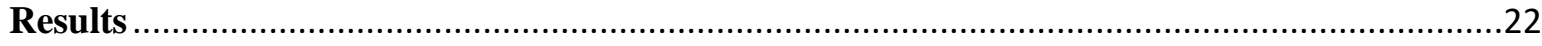

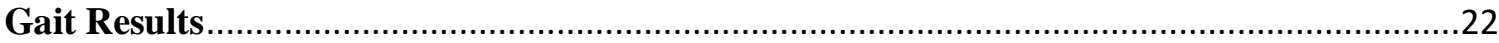

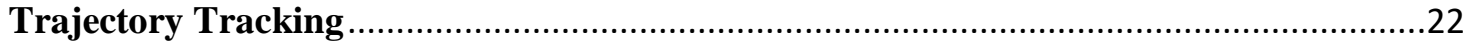

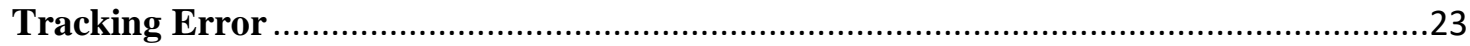

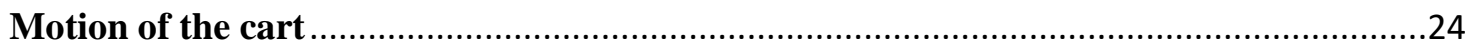

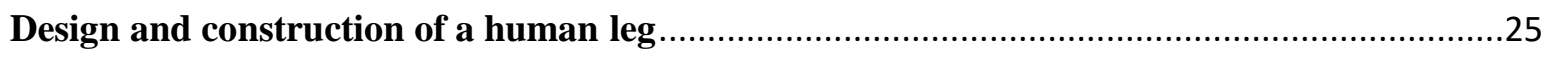

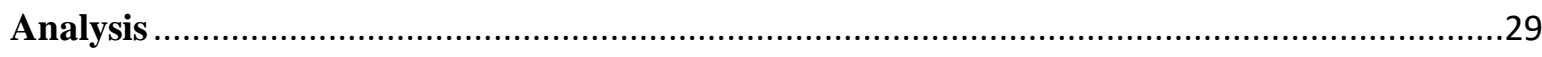

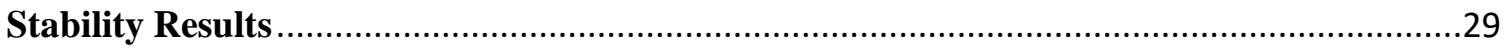

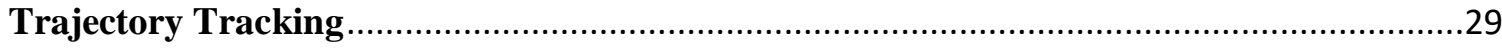

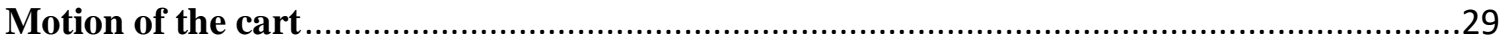

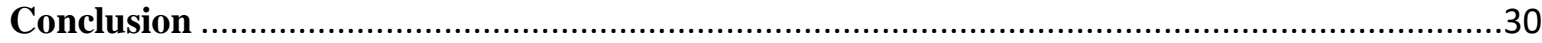

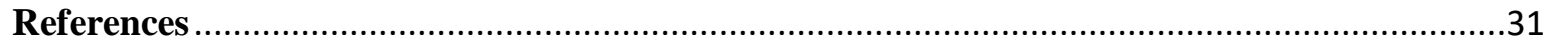

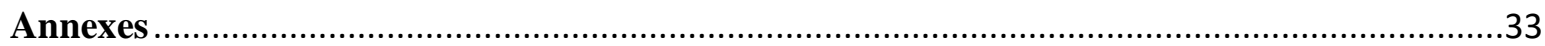

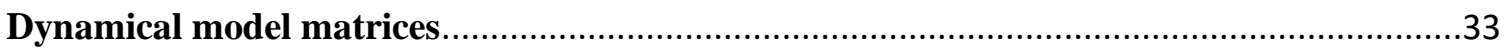

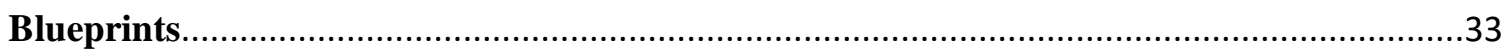




\section{Figure table}

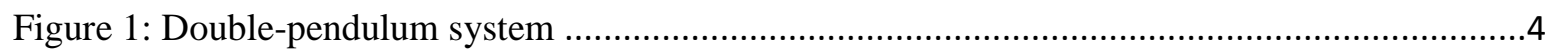

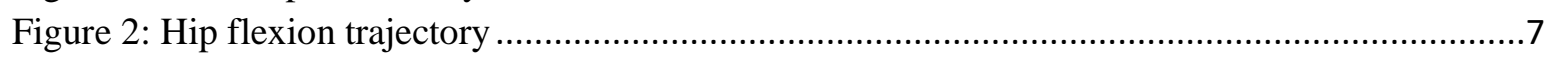

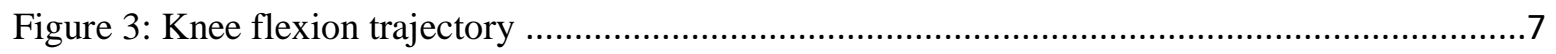

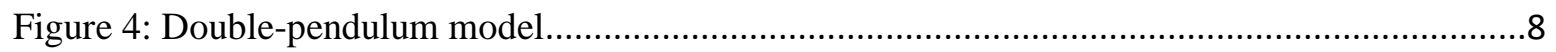

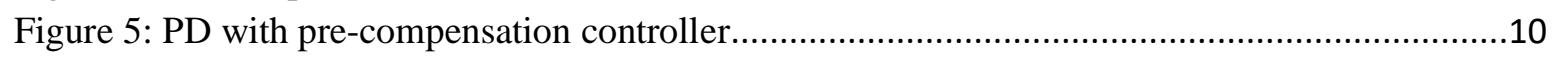

Figure 6: Trajectories for the stability test...........................................................................

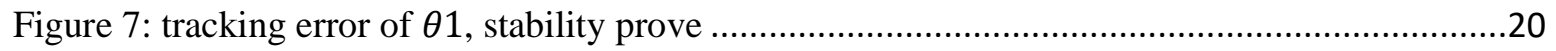

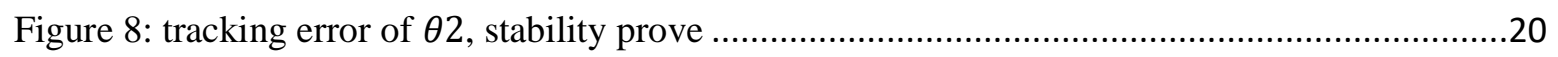

Figure 9: Table of Body Segment Inertial Properties (BSIP's) ...............................................21

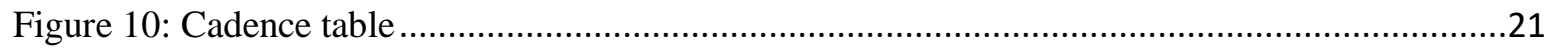

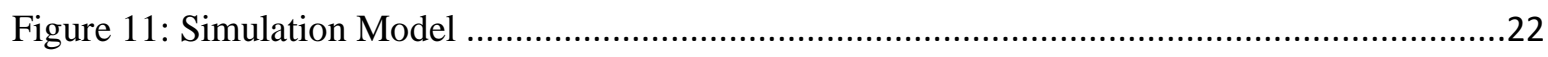

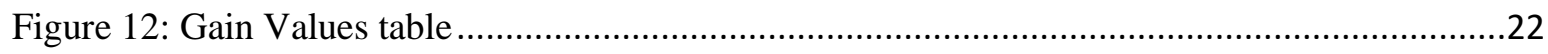

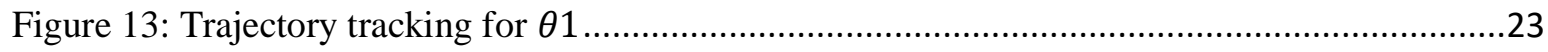

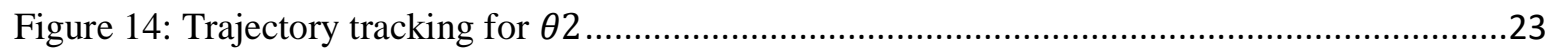

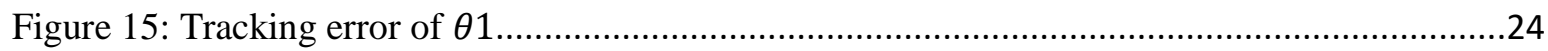

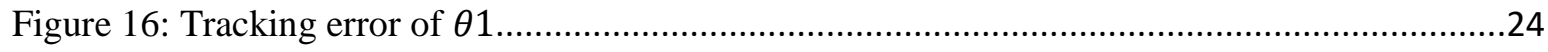

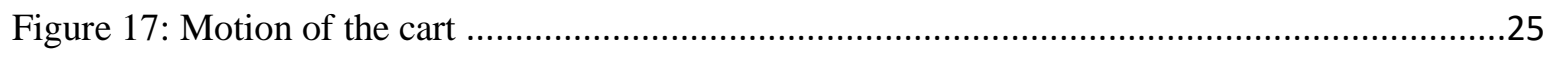

Figure 18: Locations of selected anatomical landmarks and SCSs ..........................................26

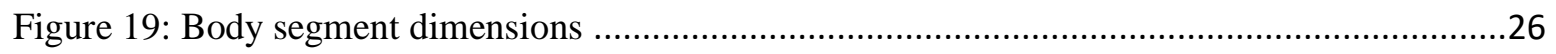

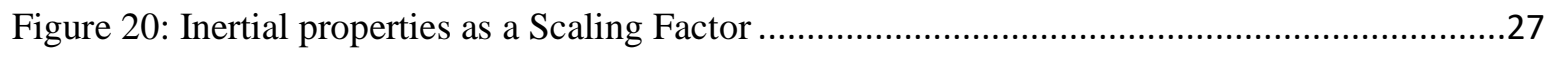

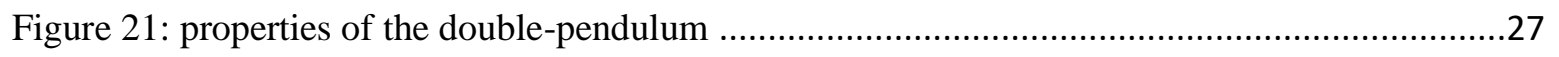

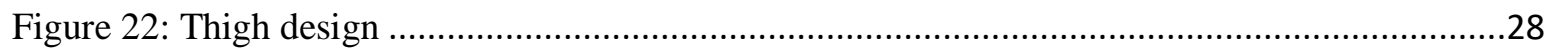

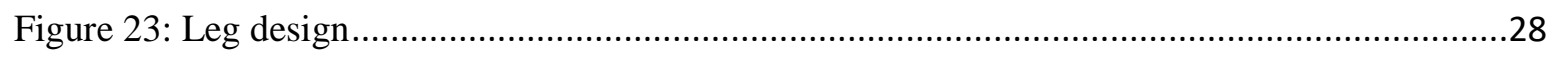

Figure 24: Assembly of the leg and the Stewart platform......................................................28 


\section{Introduction}

This project deals with the study of a double-pendulum cart system for the potential application in rehabilitation robotics, specifically in the rehabilitation of the lower limbs due to fracture. The objective is to perform a passive rehabilitation of the gait without forcing the limbs (thigh and leg), but instead encouraging the hip to achieve the proper motion of the lower limbs.

Although the approach is new, the rehabilitation robotics in lower limbs has been studied extensively, because due the precise and cyclic task that is walking, a machine can able to help in this process of therapy. One robot used in different rehabilitation robotics projects is the Stewart platform, giving many degrees of freedom (DOF) to work on patients, [1] designs a Stewart platform that provides a desired pure rotational motion required in the rehabilitation of the foot for patients with neuropathy, [2] is a similar work, using this platform as an anklefoot assist device for rehabilitation. Other projects involve the construction of exoskeletons to go along with the motion of the patient [3].

Based on the intention to generate a more passive rehabilitation therapy, a new approach of the double pendulum is proposed.

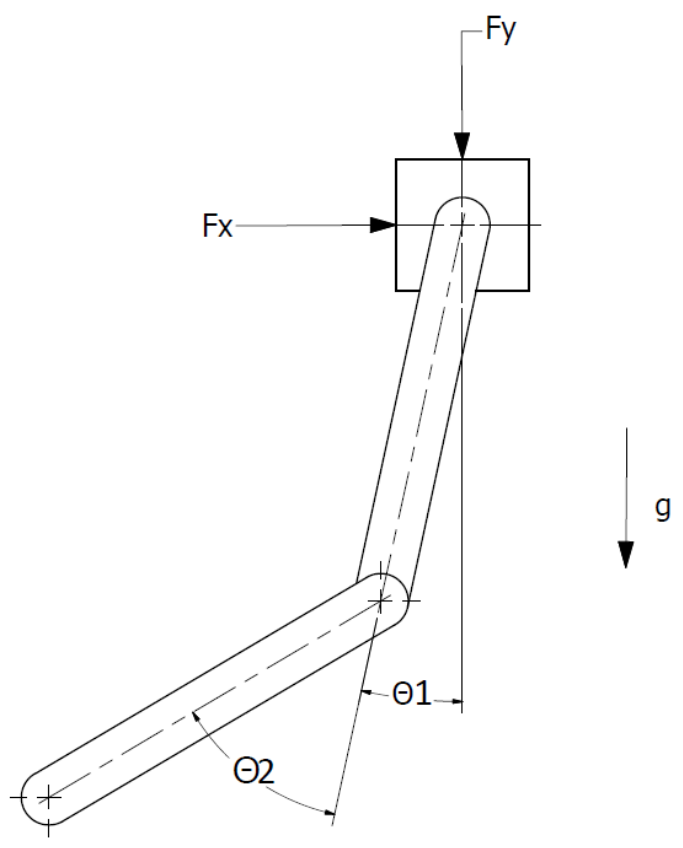

Figure 1: Double-pendulum system 
The model shown in figure 1 is a 2 DOF system with two force entries in the cart $\left(F_{x} F_{y}\right)$ and two outputs $\theta_{1}$ and $\theta_{2}$ and as seen, the gravity points downwards. To link this model to the human leg and the rehabilitation strategy, it is assumed that the cart is the hip, the first limb is the thigh and the second limb is the leg, and applying force to the hip, $\theta_{1}$ and $\theta_{2}$ move in order to achieve the gait.

This assumption of the double-pendulum as a leg is frequently used to study the human locomotion, [4] studies the a new conceptual walking model benefiting from swing leg dynamics, considering a double-pendulum equipped with combinations of biarticular springs for the swing leg. Other proposals such as [5], that models the whole walking motion as an inverted pendulum structure where the one leg locomotion is similar to Self-Impact Double Pendulum (SIDP) with a stopper at the lower-joint.

The formulation has no only a strong influence in rehabilitation robotics, but also in the research of prosthetics, [6] proposes a new control algorithm for a semi-active prosthetic knee during the swing phase, simulations using a double pendulum model shows that the generated knee trajectory is similar to the normal gait. The investigation on prosthetics also gives a better understanding of the inertial properties, stiffness and damping coefficients of the leg to stablish better models based on a double-pendulum [7].

Another application of the double-pendulum model is the potential use in legged robots, [8] studies the control for an ideal double pendulum dynamical system that places feet into ground targets for a wide range of initial conditions and in the presence of significant locomotion disturbances.

The double pendulum as stablished in figure 1 has some interesting challenges in terms of control due to its non-linear and unstable behavior, and the indirect actuation in terms of the control variables. The work related to pendulum control seeks the stabilization of the degrees of freedom in cases such as the inverted pendulum or crane systems and giving the variables will be in a close neighborhood to the stabilization point it is possible to linearize the model and therefore use conventional lineal controllers such as PID's.

The first approach of an inverted-pendulum control is a PID, as in this paper [9], PID controllers are applied to the stabilization and tracking control of three types of inverted pendulum. [10] studies similar inverted-pendulums but found that the LQR controller is better than the conventional PID, but both can track properly the trajectory.

A real example of the use of this model is the industrial cranes, that commonly suffer from effects caused by the so-called double-pendulum phenomenon. Many researches have presented models to propose controllers, in this paper [11] presents the control problem of a double pendulum crane system and propose a time-optimal trajectory planning method with the consideration of various constraints which can achieve the objectives of both accurate trolley positioning and double pendulum swing suppression. [12] shows a different approach 
to the stabilization of industrial cranes, using a nonlinear controller, they present an adaptive ant swing control strategy for crane systems with double-pendulum swing effects and position with reduced overshoots and effectively eliminate the double-pendulum swing angles at the same time.

For this project a new control model for a double-pendulum system is presented. The control is formed by a non-linear part, which is the pre-compensated dynamics and a linear part which is the closed-loop control. Some similar structures are used in control research, [13] uses a fuzzy pre-compensated with a PI speed controller for permanent magnet brushless DC motor drive.

Other non-linear controllers such as feedback linearization are used in pendulum control, [14] considers a self-impact constraint in double pendulum modeling at the knee joint and due to complex nonlinear terms in dynamics equations of the joint self-impact swing leg, they propose to design the controller by taking advantage of the feedback linearization control method. [15] studies a spatial double inverted pendulum actuated at the hip, like the model shown here, but with torques and as an inverted pendulum, this paper uses a hybrid approach where an energy-shaping, passivity-based swing-up controller hands off the control to a linear-quadratic-regulator in the vicinity of the unstable upright equilibrium.

The study presented in this project is intended to support the use of rehabilitation robotics in passive therapy, promoting the leg motion from the hip. This motivates the use of the human gait cycle as the trajectory goal for the double pendulum. Further information about this trajectory is presented in other section.

This document is organized as follows, First, we present the human gait cycle and the reference trajectories for the double pendulum. Then, we derived the dynamic model using Lagrange formulation for the trajectory tracking including friction in the joints, followed by the control model proposed in this project, a pre-compensated + PD controller, schematics are shown. A mathematical stability prove is performed using the direct method of Lyapunov and equations for the tuning of the controller are presented. Simulation parameters are shown after, which describe different configurations for the simulations performed, as well as the model in Simulink, Simmechanics is used for the simulation. Results of the trajectory tracking and tracking error are presented, motion of the cart is shown for different cadence gaits. A stability prove using the simulation is performed with tuned values of the mathematical stability prove, also a comparison with other gain values is made, different trajectories are used for these simulations. A section with the construction of the leg is made. Analysis of every section of the results are made, conclusions of the project are presented, as well as the future work and possible improvements. 


\section{Gait Analysis}

Gait analysis is the study of human locomotion, covering kinematics and kinetics of the different limbs of the body. In figure 2 and 3 are presented the graphics of the trajectories of the normalized gait. Positive values are as shown in the figure 1 . In figure 2 it is possible to see the strong deceleration in the swing phase, making the control in that part more complex.

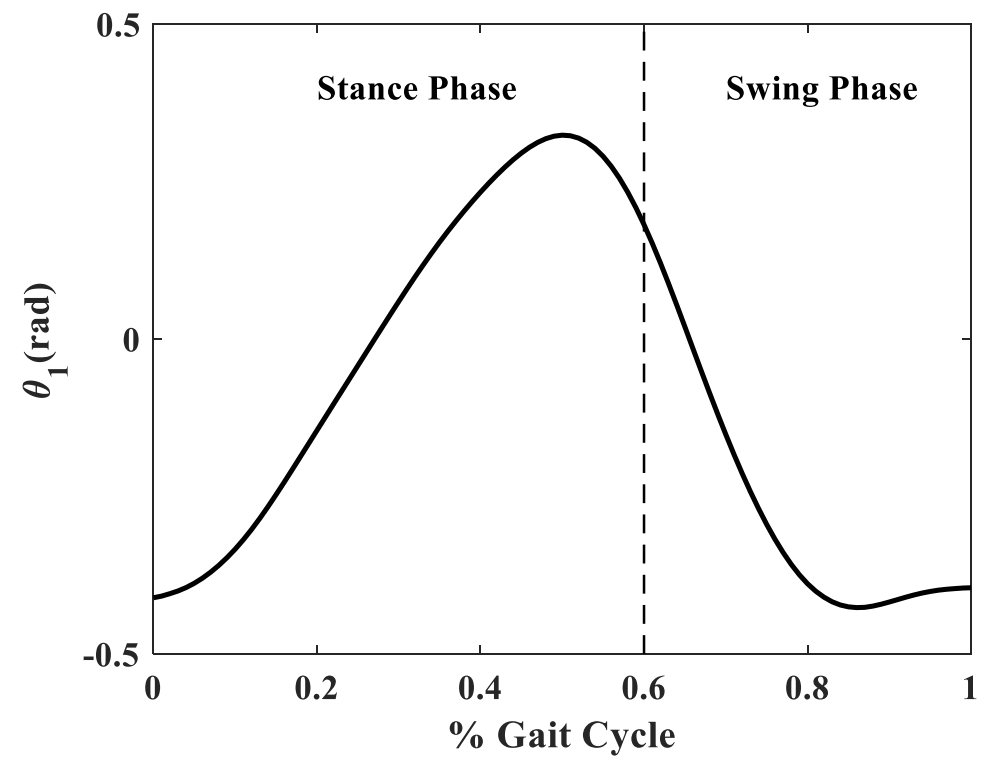

Figure 2: Hip flexion trajectory

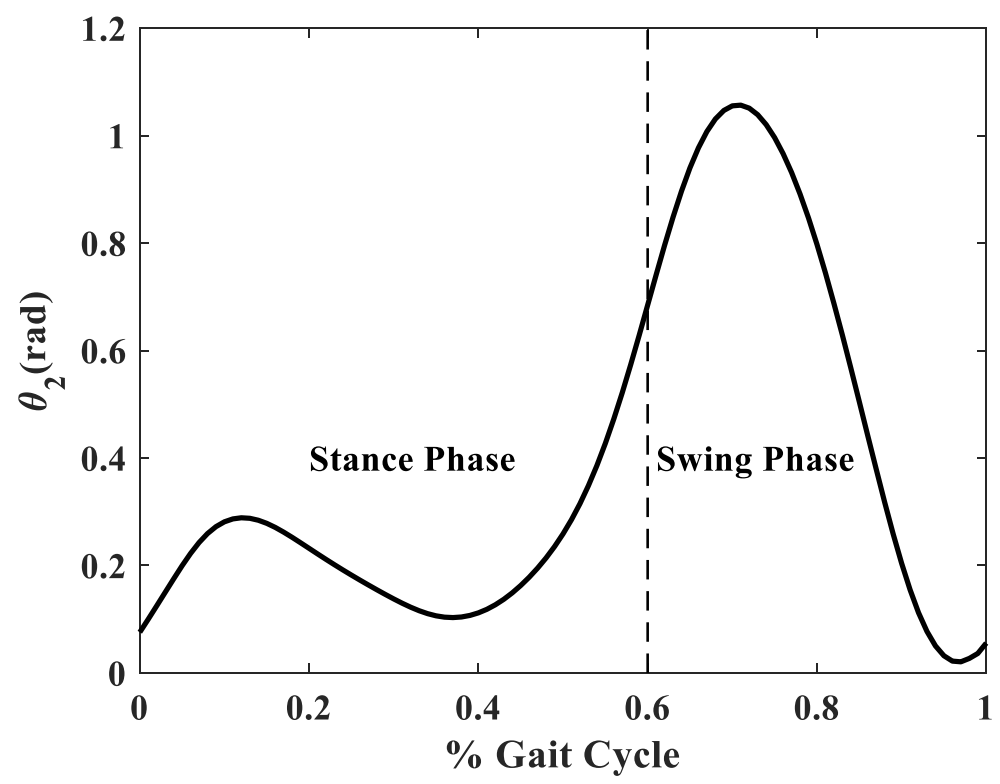

Figure 3: Knee flexion trajectory 
The trajectories followed by the hip and the knee are presented in Fig. 2 and Fig. 3 (taken from [16]). The trajectory of the leg can be divided in two phases: Stance phase and Swing phase. The first one is when the foot is in contact with the floor and covers around $60 \%$ percent of the gait cycle, the second is when the foot is not in contact with the floor and covers around $40 \%$ of the gait cycle. [17]. For this study only the swing phase is considered, because in the stance phase a different model has to be used.

\section{Double-pendulum Dynamics}

The double-pendulum under study is a two DOF system with two inputs (forces $F_{x}$ and $F_{y}$ ) and two outputs (angles $\theta_{1}$ and $\theta_{2}$ ), which are the variables to control (Fig 4). The pendulum can move in the $\mathrm{x}-\mathrm{y}$ space in order to control a given trajectory in each joint.

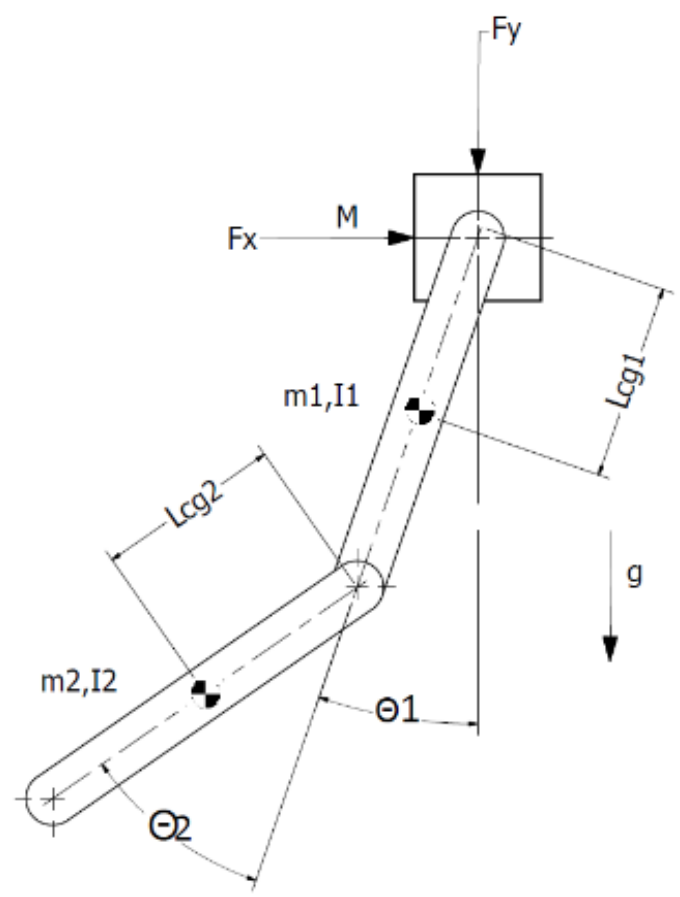

Figure 4: Double-pendulum model

The dynamic model is derived using the Lagrange formulation. Forces are positive in the directions shown, and gravity points downwards. The kinetic and potential energy of the system are:

$$
\begin{gathered}
T=\frac{1}{2} M v_{M}^{2}+\frac{1}{2} m_{1} v_{1}^{2}+\frac{1}{2} m_{2} v_{2}^{2}+\frac{1}{2} I_{1} \dot{\theta}_{1}^{2}+\frac{1}{2} I_{2}{\dot{\theta_{12}}}^{2} \\
V=-m_{1} g\left(l_{1 G} c_{1}+y\right)-m_{2} g\left(l_{2 G} c_{12}+l_{1} c_{1}+y\right)-M g y
\end{gathered}
$$


where $l_{i G}$ is the distance to the center of gravity of the corresponding segment. Then the Lagrangian is computed as:

$$
\mathcal{L}=T-V
$$

and the formulation for the hip angle $\left(\theta_{1}\right)$, knee angle $\left(\theta_{2}\right)$, the horizontal and vertical motion of the cart ( $x$ and $y$ ) can be computed as

$$
\frac{d}{d t}\left[\frac{\partial \mathcal{L}(\boldsymbol{q}, \dot{\boldsymbol{q}})}{\partial \dot{q}_{\iota}}\right]-\frac{\partial \mathcal{L}(\boldsymbol{q}, \dot{\boldsymbol{q}})}{\partial q_{i}}=Q_{i}
$$

Although there are four equations, only two DOF are actuated, the other two have an associated viscous friction, so the generalized forces $Q_{i}(i=1,2,3,4)$ are:

$$
\begin{gathered}
Q_{1}=-d_{1} \dot{\theta_{1}} \\
Q_{2}=-d_{2} \dot{\theta_{2}} \\
Q_{3}=F_{x} \\
Q_{4}=F_{y}
\end{gathered}
$$

Now, applying equation (4) to the Lagrangian the generalized forces can be obtained. The equations of the dynamics of the double-pendulum cart are:

$$
\begin{aligned}
& Q_{\theta_{1}}=I_{1} \ddot{\theta}_{1}+I_{2} \ddot{\theta}_{12}+\left(l_{1}^{2} m_{2}+l_{1 G}^{2} m_{1}\right) \ddot{\theta}_{1}+m_{2} l_{2 G}^{2} \ddot{\theta}_{12}+m_{2} g l_{2 G} s_{12} \\
& \quad-m_{2} l_{2 G}\left(\ddot{x} c_{12}+\ddot{y} s_{12}\right)+g l_{1} s_{1}\left(m_{2} l_{1}+m_{1} l_{1 G}\right) \\
& +\left(-\ddot{x} c_{1}-\ddot{y} s_{1}\right)\left(m_{2} l_{1}+m_{1} l_{1 G}\right)-m_{2} l_{1} l_{2 G} \dot{\theta}_{2}^{2} s_{2}+m_{2} l_{1} l_{2 G} c_{2}\left(2 \ddot{\theta}_{1}+\ddot{\theta_{2}}\right) \\
& \quad-2 m_{2} l_{1} l_{2 G} \dot{\theta_{1}} \dot{\theta}_{2} s_{2}
\end{aligned}
$$




$$
\begin{aligned}
Q_{y}=M \ddot{y}-g( & \left(M+m_{1}+m_{2}\right)-m_{1}\left(l_{1 G} c_{1} \dot{\theta_{1}^{2}}-\ddot{y}+l_{1 G} \ddot{\theta}_{1} s_{1}\right) \\
& -m_{2}\left(l_{2 G} c_{12} \dot{\theta}_{12}^{2}-\ddot{y}+l_{1} \ddot{\theta}_{1} s_{1}+l_{1} c_{1} \dot{\theta}_{1}{ }^{2}+l_{2 G} s_{12} \ddot{\theta}_{12}\right)
\end{aligned}
$$

Where $\theta_{12}=\theta_{1}+\theta_{2}$ and the trigonometrical function conventions are $c_{i}=\cos \left(\theta_{i}\right)$, $s_{i}=\sin \left(\theta_{i}\right) s_{12}=\sin \left(\theta_{1}+\theta_{2}\right), c_{12}=\cos \left(\theta_{1}+\theta_{2}\right)$.

Equations 9 to 12 have to be rewritten to eliminate indirect variables. Remind that the actuated variables are the forces and the controlled trajectories are $\theta_{1}$ and $\theta_{2}$. These equations can be expressed as:

$$
[M(\boldsymbol{\theta})] \ddot{\boldsymbol{\theta}}+[C(\boldsymbol{\theta}, \dot{\boldsymbol{\theta}})] \dot{\boldsymbol{\theta}}+\boldsymbol{g}(\boldsymbol{\theta})=\boldsymbol{F}
$$

In that form, the controlled variables are function of the input forces and the indirect variables (x and y) do not appear. Equation 13 is a matrix expression where: $M(\theta)$ and $C(\theta)$ are matrices $2 \times 2$ and $\dot{\boldsymbol{\theta}}_{\mathbf{1}}, \ddot{\boldsymbol{\theta}_{2}}$ and $\boldsymbol{g}(\boldsymbol{\theta})$ are vectors. $\mathbf{F}$ is a column vector $\left[F_{x}, F_{y}\right]$. The detailed matrices are presented in the annex.

\section{Control Model}

For this application a PD with pre-compensation controller is used [18]. The pre-compensation part uses the inverse-dynamics of the model to cancel the dynamics of the system. The PD part of the controller is used as a feedback controller to deal with the real following error. The control law is expressed as:

$$
\boldsymbol{F}=K_{p} \widetilde{\boldsymbol{q}}+K_{v} \widetilde{\boldsymbol{q}}+M\left(\boldsymbol{q}_{\boldsymbol{d}}\right) \ddot{\boldsymbol{q}}_{d}+C\left(\boldsymbol{q}_{\boldsymbol{d}}, \dot{\boldsymbol{q}}_{\boldsymbol{d}}\right) \dot{\boldsymbol{q}}_{\boldsymbol{d}}+g\left(\boldsymbol{q}_{\boldsymbol{d}}\right)
$$

where $K_{p}, K_{v}$ are symmetrical matrices with gain values of position and velocity. $\boldsymbol{q}_{\boldsymbol{d}}$ is the desired trajectory and $\boldsymbol{q}$ is the real trajectory; $\widetilde{\boldsymbol{q}}$ is the error $\widetilde{\boldsymbol{q}}=\boldsymbol{q}_{d}-\boldsymbol{q}$. Figure 4 shows the schematic of the control:

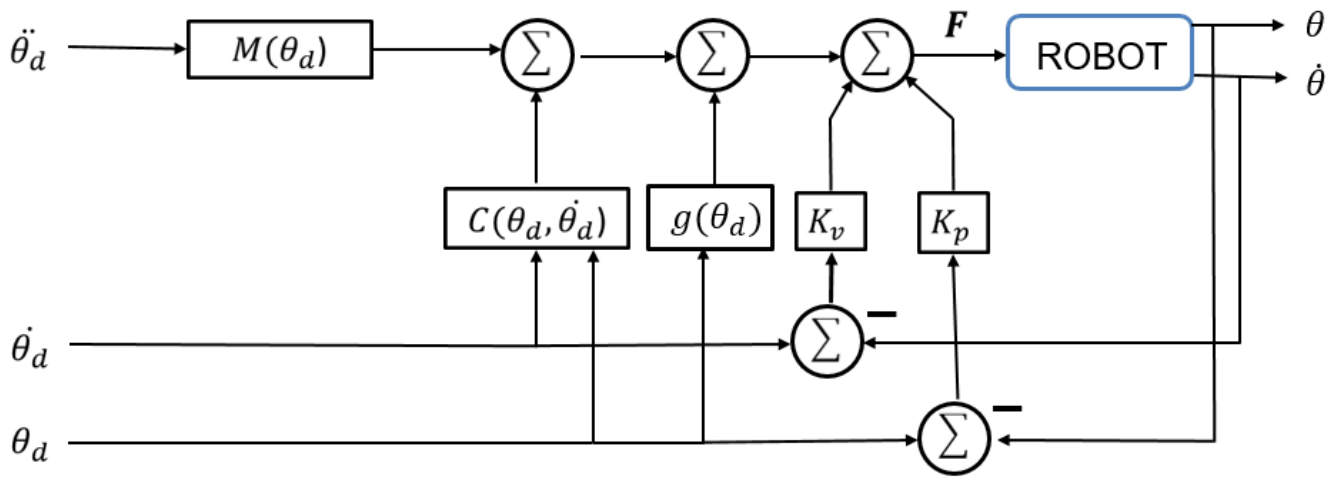

Figure 5: PD with pre-compensation controller 
The behavior of the closed loop system can be obtained substituting the force vector $\mathbf{F}$ by the model of the robot:

$$
M(\boldsymbol{q}) \ddot{\boldsymbol{q}}+C(\boldsymbol{q}, \dot{\boldsymbol{q}}) \dot{\boldsymbol{q}}+\boldsymbol{g}(\boldsymbol{q})=K_{p} \widetilde{\boldsymbol{q}}+K_{v} \widetilde{\boldsymbol{q}}+M\left(\boldsymbol{q}_{\boldsymbol{d}}\right) \ddot{\boldsymbol{q}}_{d}+C\left(\boldsymbol{q}_{\boldsymbol{d}}, \boldsymbol{q}_{\boldsymbol{d}}\right) \dot{\boldsymbol{q}}_{\boldsymbol{d}}+g\left(\boldsymbol{q}_{\boldsymbol{d}}\right)
$$

And expressing equation (15) in terms of the state vector $[\widetilde{\boldsymbol{q}} \dot{\tilde{\boldsymbol{q}}}]^{T}$

$$
\frac{d}{d t}\left[\begin{array}{c}
\widetilde{\boldsymbol{q}} \\
\dot{\widetilde{\boldsymbol{q}}}
\end{array}\right]=\left[\begin{array}{c}
\dot{\widetilde{\boldsymbol{q}}} \\
M(\boldsymbol{q})^{-1}\left[-K_{p} \widetilde{\boldsymbol{q}}-K_{v} \dot{\tilde{\boldsymbol{q}}}-C(\boldsymbol{q}, \dot{\boldsymbol{q}}) \dot{\widetilde{\boldsymbol{q}}}-\boldsymbol{h}(\widetilde{\boldsymbol{q}}, \dot{\tilde{\boldsymbol{q}}})\right.
\end{array}\right]
$$

\section{Stability Prove}

First, we define vector $\boldsymbol{q}$ as a vector of $\left[\theta_{1}, \theta_{2}\right]$

The equation 17 presents the dynamical system of 2 DOF defined in the range $(-\infty, \infty)-$ $\left\{\theta_{2} \mid \sin \theta_{2}=0\right\}$

$$
\boldsymbol{F}=K_{p} \widetilde{\boldsymbol{q}}+K_{v} \dot{\widetilde{\boldsymbol{q}}}+\left[M\left(\boldsymbol{q}_{\boldsymbol{d}}\right)\right] \ddot{\boldsymbol{q}_{\boldsymbol{d}}}+\left[C\left(\boldsymbol{q}_{\boldsymbol{d}}, \boldsymbol{q}_{\boldsymbol{d}}\right)\right] \dot{\boldsymbol{q}}_{\boldsymbol{d}}+\boldsymbol{g}\left(\boldsymbol{q}_{\boldsymbol{d}}\right)
$$

Where $K_{p}, K_{v} \in R^{2 \times 2}$ are symmetrical positive definite matrices called speed and position gains. We define $\boldsymbol{q}_{\boldsymbol{d}}$ as a vector with the desired trajectories of each control variable. The tracking error is $\widetilde{\boldsymbol{q}}=\boldsymbol{q}_{\boldsymbol{d}}-\boldsymbol{q}$, with $\boldsymbol{q}$ the vector of the real trajectories of the system.

Now we describe the corresponding variables to the dynamics of the system, but before it is important to say that the $\boldsymbol{q}_{\boldsymbol{d}}$ vector is formed by just angular positions. The inertia matrix $M\left(\boldsymbol{q}_{\boldsymbol{d}}\right)$ is defined and non-symmetrical for $\left\{\theta_{2} \mid \sin \theta_{2} \neq 0\right\} 2 \times 2$ whose elements are functions of $\boldsymbol{q}$ and its invertible.

$$
\begin{aligned}
& \operatorname{det} M\left(q_{d}\right) \\
& =\frac{\left(f_{1}\left(I_{1}, l_{2}, m_{1}, m_{2}, M, l_{1}, l_{1 G}, l_{2}\right)+\sin ^{2} q_{d}(2) \cdot f_{2}\left(I_{1}, l_{2}, m_{1}, m_{2}, M, l_{1}, l_{1 G}, l_{2}\right)\right)}{m_{2} l_{2 G} \sin q_{d}(2)\left(l_{1} m_{2}+l_{1 G} m_{1}\right)} \\
& \neq 0
\end{aligned}
$$

It is true that $\lambda_{\min }\{M\}>0$, where $\lambda_{\min }\{M\}$ is the smaller eigenvalue of the matrix $\mathrm{M}$ The closed-loop equation is

$$
\frac{d}{d t}\left[\begin{array}{c}
\widetilde{\boldsymbol{q}} \\
\dot{\widetilde{\boldsymbol{q}}}
\end{array}\right]=\left[\begin{array}{c}
\dot{\widetilde{\boldsymbol{q}}} \\
M(\boldsymbol{q})^{-1}\left[-K_{p} \widetilde{\boldsymbol{q}}-K_{v} \dot{\tilde{\boldsymbol{q}}}-C(\boldsymbol{q}, \dot{\boldsymbol{q}}) \dot{\widetilde{\boldsymbol{q}}}-\boldsymbol{h}(\widetilde{\boldsymbol{q}}, \dot{\widetilde{\boldsymbol{q}}})\right.
\end{array}\right]
$$


Where

$$
\begin{gathered}
\boldsymbol{h}(\widetilde{\boldsymbol{q}}, \dot{\tilde{\boldsymbol{q}}})=\left[M\left(\boldsymbol{q}_{\boldsymbol{d}}\right)-M\left(\boldsymbol{q}_{\boldsymbol{d}}-\widetilde{\boldsymbol{q}}\right)\right] \ddot{\boldsymbol{q}}_{\boldsymbol{d}}+\left[C\left(\boldsymbol{q}_{\boldsymbol{d}}, \boldsymbol{q}_{\boldsymbol{d}}\right)-C\left(\boldsymbol{q}_{\boldsymbol{d}}-\widetilde{\boldsymbol{q}}, \boldsymbol{q}_{\boldsymbol{d}}-\dot{\tilde{\boldsymbol{q}}}\right)\right] \dot{\boldsymbol{q}}_{\boldsymbol{d}}+\boldsymbol{g}\left(\boldsymbol{q}_{\boldsymbol{d}}\right) \\
-\boldsymbol{g}\left(\boldsymbol{q}_{\boldsymbol{d}}-\widetilde{\boldsymbol{q}}\right)
\end{gathered}
$$

\section{Unique Equilibrium}

To prove that the origin is an equilibrium, $\left[\begin{array}{ll}\widetilde{\boldsymbol{q}}^{T} & \dot{\widetilde{\boldsymbol{q}}}^{T}\end{array}\right]^{T}=\mathbf{0}$ is evaluated

It is easy to that the equation 19 is reduced to the solution vectors $\left[\begin{array}{ll}\widetilde{\boldsymbol{q}}^{T} & \dot{\widetilde{\boldsymbol{q}}}^{T}\end{array}\right]^{T}=\left[\begin{array}{ll}\boldsymbol{S}^{T} & \mathbf{0}^{T}\end{array}\right]^{T}$, being $\mathbf{s}$ a solution of the equation

$$
K_{p} \boldsymbol{s}+\boldsymbol{h}(\boldsymbol{s}, \mathbf{0})=\mathbf{0}
$$

This equation satisfies the trivial solution $\mathbf{s}=\mathbf{0}$, but we need to prove uniqueness in the equilibrium, so it is necessary to assure certain conditions in the $K_{p}$ matrix. It is defined

$$
\boldsymbol{k}(\boldsymbol{s})=K_{p}^{-1} \boldsymbol{h}(\boldsymbol{s}, \mathbf{0})
$$

Any fixed-point $\mathbf{s}$ is a solution of the equation 21 , and therefore we need to fin the conditions on $K_{p}$ so that $\boldsymbol{k}(\boldsymbol{s})$ has a unique fixed-point. And knowing that the equation guarantees the trivial solution, that will be the only one.

First notice that for every vector $\boldsymbol{x}, \boldsymbol{y} \in R^{n}$ satisfices that

$$
\|\boldsymbol{k}(\boldsymbol{x})-\boldsymbol{k}(\boldsymbol{y})\| \leq\left\|K_{p}^{-1}[\boldsymbol{h}(\boldsymbol{x}, \mathbf{0})-\boldsymbol{h}(\boldsymbol{y}, \mathbf{0})]\right\|
$$

And $K_{p}$ is a diagonal matrix satisfies that

$$
\|\boldsymbol{k}(\boldsymbol{x})-\boldsymbol{k}(\boldsymbol{y})\| \leq \lambda_{\operatorname{Max}}\left\{K_{p}^{-1}\right\}\|\boldsymbol{h}(\boldsymbol{x}, \mathbf{0})-\boldsymbol{h}(\boldsymbol{y}, \mathbf{0})\|
$$

One of the characteristics of the residual dynamics $\boldsymbol{h}(\widetilde{\boldsymbol{q}}, \dot{\tilde{\boldsymbol{q}}})$ is that it does not grow faster than $\|\widetilde{\boldsymbol{q}}\|$ and $\|\dot{\tilde{\boldsymbol{q}}}\|$. The norm of the residual dynamics satisfies that

$$
\begin{gathered}
\|\boldsymbol{h}(\boldsymbol{x}, \mathbf{0})-\boldsymbol{h}(\boldsymbol{y}, \mathbf{0})\| \leq\left\|\left[M\left(\boldsymbol{q}_{\boldsymbol{d}}-\boldsymbol{y}\right)-M\left(\boldsymbol{q}_{\boldsymbol{d}}-\boldsymbol{x}\right)\right] \ddot{\boldsymbol{q}}_{\boldsymbol{d}}\right\| \\
+\left\|\left[C\left(\boldsymbol{q}_{\boldsymbol{d}}-\boldsymbol{y}, \boldsymbol{q}_{\boldsymbol{d}}\right)-C\left(\boldsymbol{q}_{\boldsymbol{d}}-\boldsymbol{x}, \boldsymbol{q}_{\boldsymbol{d}}\right)\right] \boldsymbol{q}_{\boldsymbol{d}}\right\|+\left\|\boldsymbol{g}\left(\boldsymbol{q}_{\boldsymbol{d}}-\boldsymbol{y}\right)-\boldsymbol{g}\left(\boldsymbol{q}_{\boldsymbol{d}}-\boldsymbol{x}\right)\right\|
\end{gathered}
$$

\section{Matrix properties of the dynamics model}

The properties of the inertia and centrifugal matrices and gravitational term are defined 


$$
\begin{gathered}
\|M(\boldsymbol{x}) \mathbf{z}-M(\boldsymbol{y}) \boldsymbol{z}\| \leq k_{M}\|\boldsymbol{x}-\boldsymbol{y}\|\|\boldsymbol{z}\| \\
\|C(\boldsymbol{x}, \mathbf{z}) \boldsymbol{\omega}-C(\boldsymbol{y}, \boldsymbol{v}) \boldsymbol{\omega}\| \leq k_{C 1}\|\boldsymbol{z}-\boldsymbol{v}\|\|\boldsymbol{\omega}\|+k_{C 2}\|\boldsymbol{z}\|\|\boldsymbol{x}-\boldsymbol{y}\|\|\boldsymbol{\omega}\| \\
\|\boldsymbol{g}(\boldsymbol{x})-\boldsymbol{g}(\boldsymbol{y})\| \leq k_{g}\|\boldsymbol{x}-\boldsymbol{y}\|
\end{gathered}
$$

Where $k_{M}, k_{C 1}, k_{C 2}$ are scalars greater than zero.

The prove of the properties are in the reference [18], appendix C, properties 4.2.3, 4.3.6 and 4.4.3. In general, the next theorem is used:

Consider the vector $\boldsymbol{x} \in R^{n}$ so, the Euclidian norm $\|\boldsymbol{x}\|$ satisfies

$$
\|x\| \leq n\left[\max \left\{\left|x_{i}\right|\right\}\right]
$$

Applying the previous properties to equation 24 we get

$$
\|\boldsymbol{h}(\boldsymbol{x}, \mathbf{0})-\boldsymbol{h}(\boldsymbol{y}, \mathbf{0})\| \leq\left[k_{g}+k_{M}\left\|\ddot{\boldsymbol{q}}_{\boldsymbol{d}}\right\|_{M}+k_{C 2}\left\|\dot{\boldsymbol{q}}_{\boldsymbol{d}}\right\|_{M}^{2}\right]\|\boldsymbol{x}-\boldsymbol{y}\|
$$

Using $\lambda_{\operatorname{Max}}\left\{K_{p}^{-1}\right\}=1 / \lambda_{\min }\left\{K_{p}\right\}$ and replacing the equation 28 in equation 23 we get

$$
\|\boldsymbol{k}(\boldsymbol{x})-\boldsymbol{k}(\boldsymbol{y})\| \leq \frac{1}{\lambda_{\min }\left\{K_{p}\right\}}\left[k_{g}+k_{M}\left\|\ddot{\boldsymbol{q}}_{\boldsymbol{d}}\right\|_{M}+k_{C 2}\left\|\dot{\boldsymbol{q}}_{\boldsymbol{d}}\right\|_{M}^{2}\right]\|\boldsymbol{x}-\boldsymbol{y}\|
$$

To assure that the equilibrium is unique, the map contraction theorem is used:

Let $\Omega \subset R^{m}$, consider the next continuous function

$$
\begin{gathered}
\boldsymbol{f}: R^{n} \times \Omega \rightarrow R^{n} \\
{\left[\begin{array}{l}
\boldsymbol{x} \\
\boldsymbol{\theta}
\end{array}\right] \rightarrow \boldsymbol{f}(\boldsymbol{x}, \boldsymbol{\theta})}
\end{gathered}
$$

Suppose that there is a constant non-negative $k$ such as for every $\boldsymbol{x}, \boldsymbol{y} \in \boldsymbol{R}^{n}$ and $\boldsymbol{\theta} \in \Omega$ has to

$$
\|\boldsymbol{f}(\boldsymbol{x}, \boldsymbol{\theta})-\boldsymbol{f}(\boldsymbol{y}, \boldsymbol{\theta})\| \leq k\|\boldsymbol{x}-\boldsymbol{y}\|
$$

If the constant $k$ is strictly minor than one, then for any $\boldsymbol{\theta} \in \Omega$, it satisfies that the function $\boldsymbol{f}(\boldsymbol{x}, \boldsymbol{\theta})$ has a unique fixed-point $\boldsymbol{x}^{*} \in R^{n}$

Therefore, we got to assure

$$
\frac{1}{\lambda_{\min }\left\{K_{p}\right\}}\left[k_{g}+k_{M}\left\|\ddot{\boldsymbol{q}}_{\boldsymbol{d}}\right\|_{M}+k_{C 2}\left\|\boldsymbol{q}_{\boldsymbol{d}}\right\|_{M}^{2}\right]<1
$$

So, the condition for $\boldsymbol{k}(\boldsymbol{s})$ has a unique fixed-point of the closed-loop system is

$$
\lambda_{\min }\left\{K_{p}\right\}>k_{g}+k_{M}\left\|\ddot{\boldsymbol{q}}_{\boldsymbol{d}}\right\|_{M}+k_{C 2}\left\|\dot{\boldsymbol{q}}_{\boldsymbol{d}}\right\|_{M}^{2}
$$




\section{Vector hyperbolic tangent function}

The vector hyperbolic tangent function is defined as

$$
\boldsymbol{f}(\boldsymbol{x})=\left[\begin{array}{c}
\tanh \left(x_{1}\right) \\
\vdots \\
\tanh \left(x_{n}\right)
\end{array}\right]
$$

This function satisfies the next properties

$$
\begin{gathered}
\|\boldsymbol{f}(\boldsymbol{x})\| \leq \alpha_{1}\|\boldsymbol{x}\|, \\
\|\boldsymbol{f}(\boldsymbol{x})\| \leq \alpha_{2}, \\
\|\boldsymbol{f}(\boldsymbol{x})\|^{2} \leq \alpha_{3} \boldsymbol{f}(\boldsymbol{x})^{T} \boldsymbol{x}, \\
\|\boldsymbol{f}(\boldsymbol{x})\| \leq \alpha_{4}\|\dot{\boldsymbol{x}}\|
\end{gathered}
$$

With $\alpha_{1}=1, \quad \alpha_{2}=\sqrt{n}, \alpha_{3}=1, \alpha_{4}=1$

Some conditions for $K_{v}$ and $K_{d}$ are defined

It is supposed a constant $\gamma>0$ and the matrix $K_{v}$ is selected big enough in the way of

And $K_{p}$ in the way of

$$
\lambda_{\text {Max }}\left\{K_{v}\right\} \geq \lambda_{\min }\left\{K_{v}\right\}>k_{h 1}+k_{C 1}+\gamma b
$$

$$
\lambda_{\text {Max }}\left\{K_{p}\right\} \geq \lambda_{\min }\left\{K_{p}\right\}>\gamma^{2} \frac{\alpha_{1}^{2} \lambda_{\text {Max }}^{2}\{M\}}{\lambda_{\min }\{M\}}
$$

In the book [18] there is information about the previous constants

\section{Lyapunov function}

The next Lyapunov function is considered

$$
V(t, \widetilde{\boldsymbol{q}}, \dot{\widetilde{\boldsymbol{q}}})=\frac{1}{2} \dot{\widetilde{\boldsymbol{q}}}^{T} M(\boldsymbol{q}) \dot{\widetilde{\boldsymbol{q}}}+\frac{1}{2} \dot{\widetilde{\boldsymbol{q}}}^{T} K_{p} \dot{\widetilde{\boldsymbol{q}}}+\gamma \boldsymbol{f}(\widetilde{\boldsymbol{q}})^{T} M(\boldsymbol{q}) \dot{\widetilde{\boldsymbol{q}}}
$$

Where $\boldsymbol{f}(\widetilde{\boldsymbol{q}})$ is the vector hyperbolic tangent function and $\gamma>0$ is a giving constant

It is possible to delimit the function to prove that is positive definite and radially uncoupled First the third sum satisfies that

$$
\begin{gathered}
-\gamma \boldsymbol{f}(\widetilde{\boldsymbol{q}})^{T} M(\boldsymbol{q}) \dot{\widetilde{\boldsymbol{q}}} \leq \gamma\|\boldsymbol{f}(\widetilde{\boldsymbol{q}})\|\|M(\boldsymbol{q}) \dot{\widetilde{\boldsymbol{q}}}\| \\
-\gamma \boldsymbol{f}(\widetilde{\boldsymbol{q}})^{T} M(\boldsymbol{q}) \dot{\widetilde{\boldsymbol{q}}} \leq \gamma \alpha_{1} \lambda_{M a x}\{M\}\|\widetilde{\boldsymbol{q}}\|\|\dot{\tilde{\boldsymbol{q}}}\|
\end{gathered}
$$




$$
\gamma \boldsymbol{f}(\widetilde{\boldsymbol{q}})^{T} M(\boldsymbol{q}) \dot{\widetilde{\boldsymbol{q}}} \geq-\gamma \alpha_{1} \lambda_{\operatorname{Max}}\{M\}\|\widetilde{\boldsymbol{q}}\|\|\dot{\tilde{\boldsymbol{q}}}\|
$$

The other two terms delimit the same way

$$
\begin{aligned}
& \frac{1}{2} \dot{\tilde{\boldsymbol{q}}}^{T} M(\boldsymbol{q}) \dot{\widetilde{\boldsymbol{q}}} \geq \frac{1}{2} \lambda_{\min }\{M\}\|\dot{\widetilde{\boldsymbol{q}}}\|^{2} \\
& \frac{1}{2} \dot{\widetilde{\boldsymbol{q}}}^{T} K_{p} \dot{\widetilde{\boldsymbol{q}}} \geq \frac{1}{2} \lambda_{\min }\{K\}\|\dot{\widetilde{\boldsymbol{q}}}\|\|\widetilde{\boldsymbol{q}}\|
\end{aligned}
$$

And written in matrixial way the function satisfies the next inequation

$$
V(t, \widetilde{\boldsymbol{q}}, \dot{\boldsymbol{q}}) \geq \frac{1}{2}\left[\begin{array}{l}
\|\widetilde{\boldsymbol{q}}\| \\
\|\tilde{\tilde{\boldsymbol{q}}}\|
\end{array}\right]^{T}\left[\begin{array}{cc}
\lambda_{\min }\left\{K_{p}\right\} & -\gamma \alpha_{1} \lambda_{M a x}\{M\} \\
-\gamma \alpha_{1} \lambda_{\operatorname{Max}}\{M\} & \lambda_{\min }\{M\}
\end{array}\right]\left[\begin{array}{l}
\|\widetilde{\boldsymbol{q}}\| \\
\|\dot{\tilde{\boldsymbol{q}}}\|
\end{array}\right]
$$

You can see that the equation is positive definite and radially uncoupled by the hypothesis made in equation 30. The function can be bounded superiorly, doing the same procedure as before

$$
V(t, \widetilde{\boldsymbol{q}}, \dot{\tilde{\boldsymbol{q}}}) \leq \frac{1}{2}\left[\begin{array}{l}
\|\widetilde{\boldsymbol{q}}\| \\
\|\dot{\boldsymbol{q}}\|
\end{array}\right]^{T}\left[\begin{array}{cc}
\lambda_{\operatorname{Max}}\left\{K_{p}\right\} & \gamma \alpha_{1} \lambda_{\operatorname{Max}}\{M\} \\
\gamma \alpha_{1} \lambda_{\operatorname{Max}}\{M\} & \lambda_{\text {Max }}\{M\}
\end{array}\right]\left[\begin{array}{l}
\|\widetilde{\boldsymbol{q}}\| \\
\|\dot{\boldsymbol{q}}\|
\end{array}\right]
$$

Being a positive definite radially uncoupled function, satisfying the conditions stablished by the constants

$$
\lambda_{\operatorname{Max}}\left\{K_{p}\right\}>\gamma^{2} \alpha_{1}^{2} \lambda_{\operatorname{Max}}\{M\}
$$

This means that function is diminishing, giving the hypothesis of the equation 30.

\section{Time derivative}

To guarantee that the global asymptotic stability exists according to Lyapunov, it satisfies that $V(t, \boldsymbol{x})$ is a function that is positive definite (globally), radially uncoupled and diminishing, such that its derivative satisfies

$$
\begin{gathered}
\dot{V}(t, \mathbf{0})=0 \quad \forall t \geq 0 \\
\dot{V}(t, \boldsymbol{x})<0 \quad \forall t \geq 0 \quad \forall \boldsymbol{x} \neq \mathbf{0} \in R^{n}
\end{gathered}
$$

Therefore, we evaluate the derivative of the equation 31. In the derivative of the next equation the term $\frac{d}{d t}(\dot{\widetilde{\boldsymbol{q}}})$ is replaced by equation 16

$$
\begin{aligned}
& \dot{V}(t, \widetilde{\boldsymbol{q}}, \dot{\widetilde{\boldsymbol{q}}})=\dot{\widetilde{\boldsymbol{q}}}^{T}\left[-K_{p} \widetilde{\boldsymbol{q}}-K_{v} \dot{\widetilde{\boldsymbol{q}}}-C(\boldsymbol{q}, \dot{\boldsymbol{q}}) \dot{\widetilde{\boldsymbol{q}}}-\boldsymbol{h}(\widetilde{\boldsymbol{q}}, \dot{\widetilde{\boldsymbol{q}}})\right]+\frac{1}{2} \dot{\tilde{\boldsymbol{q}}}^{T} \dot{M}(\boldsymbol{q}) \dot{\widetilde{\boldsymbol{q}}}^{-\dot{\boldsymbol{q}}^{T} K_{p}} \dot{\widetilde{\boldsymbol{q}}}^{\prime} \\
& +\gamma \dot{\boldsymbol{f}}(\widetilde{\boldsymbol{q}})^{T} M(\boldsymbol{q}) \dot{\tilde{\boldsymbol{q}}}+\gamma \boldsymbol{f}(\widetilde{\boldsymbol{q}})^{T} \dot{M}(\boldsymbol{q}) \dot{\widetilde{\boldsymbol{q}}} \\
& +\gamma \boldsymbol{f}(\widetilde{\boldsymbol{q}})^{T}\left[-K_{p} \widetilde{\boldsymbol{q}}-K_{v} \dot{\widetilde{\boldsymbol{q}}}-C(\boldsymbol{q}, \dot{\boldsymbol{q}}) \dot{\widetilde{\boldsymbol{q}}}-\boldsymbol{h}(\widetilde{\boldsymbol{q}}, \dot{\widetilde{\boldsymbol{q}}})\right]
\end{aligned}
$$


It is easy to see that the condition $\dot{V}(t, \mathbf{0})=0$ is met. Now it is necessary to bound equation 32 superiorly by a function strictly less than zero, in order to satisfy the second condition of the direct method of Lyapunov

$$
\begin{aligned}
\dot{V}(t, \widetilde{\boldsymbol{q}}, \dot{\overrightarrow{\boldsymbol{q}}})=- & \dot{\tilde{\boldsymbol{q}}}^{T} K_{v} \dot{\dot{\boldsymbol{q}}}-\dot{\overrightarrow{\boldsymbol{q}}}^{T} C(\boldsymbol{q}, \dot{\boldsymbol{q}}) \dot{\overrightarrow{\boldsymbol{q}}}-\dot{\tilde{\boldsymbol{q}}}^{T} \boldsymbol{h}(\widetilde{\boldsymbol{q}}, \dot{\overrightarrow{\boldsymbol{q}}})+\frac{\gamma}{2}\left(\dot{\tilde{\boldsymbol{q}}}^{T}+\boldsymbol{f}(\widetilde{\boldsymbol{q}})^{T}\right) \dot{M}(\boldsymbol{q}) \dot{\overrightarrow{\boldsymbol{q}}} \\
& +\gamma \dot{\boldsymbol{f}}(\widetilde{\boldsymbol{q}})^{T} M(\boldsymbol{q}) \dot{\widetilde{\boldsymbol{q}}}+-\gamma \boldsymbol{f}(\widetilde{\boldsymbol{q}})^{T} K_{p} \widetilde{\boldsymbol{q}}-\gamma \boldsymbol{f}(\widetilde{\boldsymbol{q}})^{T} K_{v} \dot{\overrightarrow{\boldsymbol{q}}} \\
& -\gamma \boldsymbol{f}(\widetilde{\boldsymbol{q}})^{T} C(\boldsymbol{q}, \dot{\boldsymbol{q}}) \dot{\widetilde{\boldsymbol{q}}}-\gamma \boldsymbol{f}(\widetilde{\boldsymbol{q}})^{T} \boldsymbol{h}(\widetilde{\boldsymbol{q}}, \dot{\widetilde{\boldsymbol{q}}})
\end{aligned}
$$

As before, the terms that have $K_{p}$ and $K_{v}$ can be bounded by the eigenvalues of the matrix. We also use the properties of the hyperbolic tangent function to delimit the terms that are contained

$$
\begin{gathered}
-\dot{\tilde{\boldsymbol{q}}}^{T} K_{v} \widetilde{\boldsymbol{q}} \leq-\lambda_{\min }\left\{K_{v}\right\}\|\dot{\tilde{\boldsymbol{q}}}\|^{2} \\
-\gamma \boldsymbol{f}(\widetilde{\boldsymbol{q}})^{T} K_{p} \dot{\tilde{\boldsymbol{q}}} \leq-\gamma \frac{\lambda_{\min }\left\{K_{p}\right\}}{\alpha_{3}}\|f(\widetilde{\boldsymbol{q}})\|^{2} \\
\gamma \boldsymbol{f}(\widetilde{\boldsymbol{q}})^{T} K_{p} \dot{\widetilde{\boldsymbol{q}}} \leq \gamma \lambda_{M a x}\left\{K_{v}\right\}\|\dot{\tilde{\boldsymbol{q}}}\|\|\boldsymbol{f}(\widetilde{\boldsymbol{q}})\|
\end{gathered}
$$

Now, the terms that have the centrifugal matrix have a different handling, due to the next property

For every $\boldsymbol{q}, \boldsymbol{x}, \boldsymbol{y} \in R^{n}$

$$
\|C(\boldsymbol{q}, \boldsymbol{x}) \boldsymbol{y}\| \leq k_{C 1}\|\boldsymbol{x}\|\|\boldsymbol{y}\|
$$

Applying the inequation as a function of the error and the hyperbolic tangent

$$
\gamma \boldsymbol{f}(\widetilde{\boldsymbol{q}})^{T} C(\boldsymbol{q}, \dot{\boldsymbol{q}})^{T} \dot{\widetilde{\boldsymbol{q}}} \leq \gamma k_{C 1}\|\dot{\widetilde{\boldsymbol{q}}}\|\left\|\dot{q}_{d}\right\|_{M}\|\boldsymbol{f}(\widetilde{\boldsymbol{q}})\|+\gamma k_{C 1}\|\dot{\widetilde{\boldsymbol{q}}}\|^{2}\|\boldsymbol{f}(\widetilde{\boldsymbol{q}})\|
$$

Using the properties of the hyperbolic tangent

$$
\gamma \boldsymbol{f}(\widetilde{\boldsymbol{q}})^{T} C(\boldsymbol{q}, \dot{\boldsymbol{q}})^{T} \dot{\widetilde{\boldsymbol{q}}} \leq \gamma k_{C 1}\|\dot{\widetilde{\boldsymbol{q}}}\|\left\|\boldsymbol{q}_{\boldsymbol{d}}\right\|_{M}\|\boldsymbol{f}(\widetilde{\boldsymbol{q}})\|+\gamma k_{C 1} \alpha_{2}\|\dot{\widetilde{\boldsymbol{q}}}\|^{2}
$$

The second term can be bounded as

$$
\dot{\widetilde{\boldsymbol{q}}}^{T} C(\boldsymbol{q}, \dot{\boldsymbol{q}}) \dot{\widetilde{\boldsymbol{q}}} \leq k_{C 1}\|\dot{\widetilde{\boldsymbol{q}}}\|^{2}\left\|\dot{\boldsymbol{q}}_{\boldsymbol{d}}\right\|_{M}+k_{C 1}\|\dot{\widetilde{\boldsymbol{q}}}\|^{2}
$$

To bound the terms of the residual dynamics we use the next property. Let be $k_{h 1}, k_{h 2} \geq 0$, such that the norm of the residual dynamics satisfies that

$$
\boldsymbol{h}(\widetilde{\boldsymbol{q}}, \dot{\widetilde{\boldsymbol{q}}}) \leq k_{h 1}\|\dot{\widetilde{\boldsymbol{q}}}\|+k_{h 2}\|\boldsymbol{f}(\widetilde{\boldsymbol{q}})\|
$$

For every $\widetilde{\boldsymbol{q}}, \dot{\widetilde{\boldsymbol{q}}} \in R^{n}$ where $\boldsymbol{f}(\widetilde{\boldsymbol{q}})$ is the vector hyperbolic tangent function So, terms of the residual dynamics reduce to 


$$
\begin{gathered}
-\dot{\widetilde{\boldsymbol{q}}}^{T} \boldsymbol{h}(\widetilde{\boldsymbol{q}}, \dot{\widetilde{\boldsymbol{q}}}) \leq k_{h 1}\|\dot{\tilde{\boldsymbol{q}}}\|^{2}+k_{h 2}\|\dot{\widetilde{\boldsymbol{q}}}\|\|\boldsymbol{f}(\widetilde{\boldsymbol{q}})\| \\
-\gamma \boldsymbol{f}(\widetilde{\boldsymbol{q}})^{T} \boldsymbol{h}(\widetilde{\boldsymbol{q}}, \dot{\boldsymbol{q}}) \leq \gamma k_{h 1}\|\dot{\tilde{\boldsymbol{q}}}\|\|\boldsymbol{f}(\widetilde{\boldsymbol{q}})\|+\gamma k_{h 2}\|\boldsymbol{f}(\widetilde{\boldsymbol{q}})\|^{2}
\end{gathered}
$$

The term that contains the inertia matrix is bounded using the last property of the hyperbolic tangent

$$
\gamma \dot{\boldsymbol{f}}(\widetilde{\boldsymbol{q}})^{T} M(\boldsymbol{q}) \dot{\tilde{\boldsymbol{q}}} \leq \gamma \alpha_{4} \lambda_{M a x}\|\dot{\tilde{\boldsymbol{q}}}\|^{2}
$$

Finally, the term that contains the derivative of the inertia matrix. The matrix $\dot{M}$ as $M$, is a positive definite matrix, continuous in the range of $M$, therefore it is possible to bound it like

$$
\begin{gathered}
\frac{\gamma}{2}\left(\dot{\tilde{\boldsymbol{q}}}^{T}+\boldsymbol{f}(\widetilde{\boldsymbol{q}})^{T}\right) \dot{M}(\boldsymbol{q}) \dot{\tilde{\boldsymbol{q}}} \leq \frac{\gamma}{2}\left(\|\boldsymbol{f}(\widetilde{\boldsymbol{q}})\| \lambda_{M a x}\{\dot{M}\}\|\dot{\tilde{\boldsymbol{q}}}\|+\lambda_{M a x}\{\dot{M}\}\|\dot{\tilde{\boldsymbol{q}}}\|^{2}\right) \\
\frac{\gamma}{2}\left(\dot{\widetilde{\boldsymbol{q}}}^{T}+\boldsymbol{f}(\widetilde{\boldsymbol{q}})^{T}\right) \dot{M}(\boldsymbol{q}) \dot{\widetilde{\boldsymbol{q}}} \leq \frac{\gamma}{2} \lambda_{M a x}\{\dot{M}\}\left(\|\boldsymbol{f}(\widetilde{\boldsymbol{q}})\|\|\dot{\tilde{\boldsymbol{q}}}\|+\|\dot{\tilde{\boldsymbol{q}}}\|^{2}\right)
\end{gathered}
$$

Now the inequality that must satisfy the time derivative is described

$$
\begin{aligned}
& \dot{V}(t, \widetilde{\boldsymbol{q}}, \dot{\boldsymbol{q}}) \\
& \leq-\gamma\left[\begin{array}{c}
\|\boldsymbol{f}(\widetilde{\boldsymbol{q}})\| \\
\|\tilde{\boldsymbol{q}}\|
\end{array}\right]^{T}\left[\begin{array}{cc}
\frac{\lambda_{\min }\left\{K_{p}\right\}}{\alpha_{3}}-k_{h 2} & -a-\frac{1}{\gamma} \frac{k_{h 2}}{2} \\
-a-\frac{1}{\gamma} \frac{k_{h 2}}{2} & \frac{1}{\gamma}\left[\lambda_{\min }\left\{K_{v}\right\}-k_{h 1}-k_{C 1}\right]-b
\end{array}\right]\left[\begin{array}{c}
\|\boldsymbol{f}(\widetilde{\boldsymbol{q}})\| \\
\|\tilde{\tilde{\boldsymbol{q}}}\|
\end{array}\right] \\
& a=\frac{1}{2}\left[\lambda_{\text {Max }}\left\{K_{v}\right\}+k_{C 1}\left\|\dot{q}_{d}\right\|+k_{h 1}+\frac{1}{2} \lambda_{\operatorname{Max}}\{\dot{M}\}\right] \\
& b=\alpha_{4} \lambda_{\text {Max }}\{M\}+\alpha_{2} k_{C 1}+\frac{1}{2} \lambda_{\text {Max }}\{\dot{M}\}+k_{C 1}\left\|\dot{q}_{d}\right\|_{M}
\end{aligned}
$$

To guarantee that the matrix of the equation 45 is positive definite the component $(1,1)$ must be positive, as well as its determinant

For the first condition

$$
\begin{aligned}
& \frac{\lambda_{\text {min }}\left\{K_{p}\right\}}{\alpha_{3}}-k_{h 2}>0 \\
& \lambda_{\text {min }}\left\{K_{p}\right\}>\alpha_{3} k_{h 2}
\end{aligned}
$$


Now the determinant is

$$
\frac{1}{\gamma}\left[\frac{\lambda_{\min }\left\{K_{p}\right\}}{\alpha_{3}}-k_{h 2}\right]\left[\lambda_{\min }\left\{K_{v}\right\}-k_{h 1}-k_{C 1}\right]-\left[\frac{\lambda_{\min }\left\{K_{p}\right\}}{\alpha_{3}}-k_{h 2}\right] b-\left[a+\frac{1}{\gamma} \frac{k_{h 2}}{2}\right]^{2}>0
$$

And to guarantee that it is always positive

$$
\begin{gathered}
\lambda_{\min }\left\{K_{p}\right\}>\alpha_{3}\left[\frac{\left[2 \gamma a+k_{h 2}\right]^{2}}{4 \gamma\left[\lambda_{\min }\left\{K_{v}\right\}-k_{h 1}-k_{C 1}-\gamma b\right]}+k_{h 2}\right] \\
\lambda_{\min }\left\{K_{v}\right\}>k_{h 1}+k_{C 1}+\gamma b
\end{gathered}
$$

\section{Stability Results}

A stability prove was made to assure that the proposed control can track the trajectory. Some conditions were found to guarantee that stability. The tuning of the controller is presented

$$
\lambda_{\min }\left\{K_{v}\right\}>988
$$

We choose a value of $\lambda_{\min }\left\{K_{v}\right\}=2000$ and therefore

$$
\lambda_{\min }\left\{K_{p}\right\}>9600
$$

This are the eigenvalues, but giving that is a diagonal matrix, that values are numbers that go on the matrix.

$$
\begin{aligned}
& K_{v}=\left[\begin{array}{cc}
2000 & 0 \\
0 & 2000
\end{array}\right] \\
& K_{p}=\left[\begin{array}{cc}
9600 & 0 \\
0 & 9600
\end{array}\right]
\end{aligned}
$$

Any values bigger than the previously shown can work too.

\section{Trajectories used}

To stablish a cyclic trajectory that allow us to see the behavior of the controller in time, the trajectories proposed in [18] are used. 


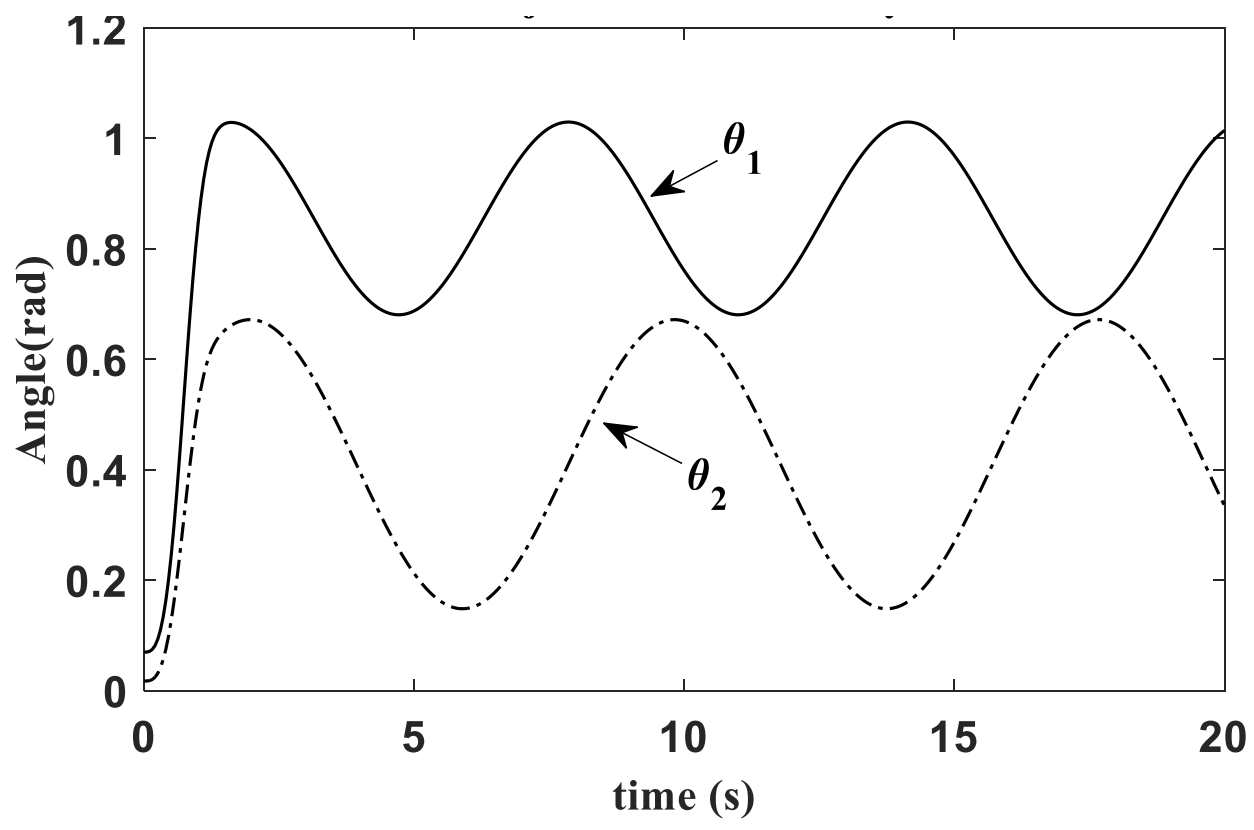

Figure 6: Trajectories for the stability test.

\section{Tracking error}

For this test, an error was induced in the initial condition and the error was evaluated. To compare with another Gain values, we present the next comparison.

\begin{tabular}{|l|l|l|l|l|}
\hline \multirow{2}{*}{$\begin{array}{l}\text { Gain } \\
\text { Values }\end{array}$} & \multicolumn{2}{|c|}{$\mathrm{K}_{\mathrm{p}}$} & \multicolumn{2}{c|}{$\mathrm{K}_{\mathrm{v}}$} \\
\cline { 2 - 5 } $\mathrm{K}_{\mathrm{p} 1}$ & $\mathrm{~K}_{\mathrm{p} 2}$ & $\mathrm{~K}_{\mathrm{v} 1}$ & $\mathrm{~K}_{\mathrm{v} 2}$ \\
\hline $\mathrm{GV}_{1}$ & 9600 & 9600 & 2000 & 2000 \\
\hline $\mathrm{GV}_{2}$ & 785 & 785 & 250 & 250 \\
\hline
\end{tabular}




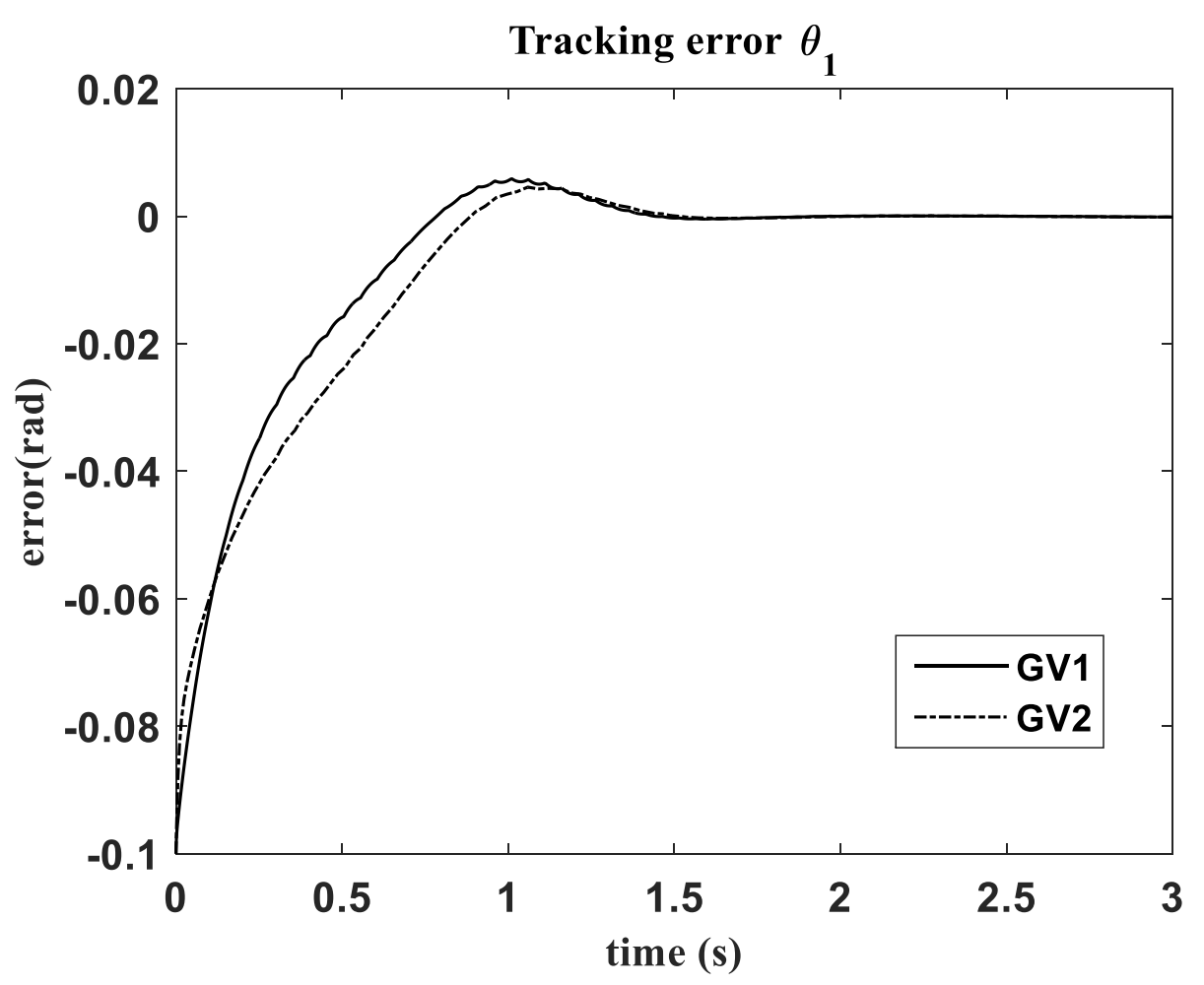

Figure 7: tracking error of $\theta_{1}$, stability prove

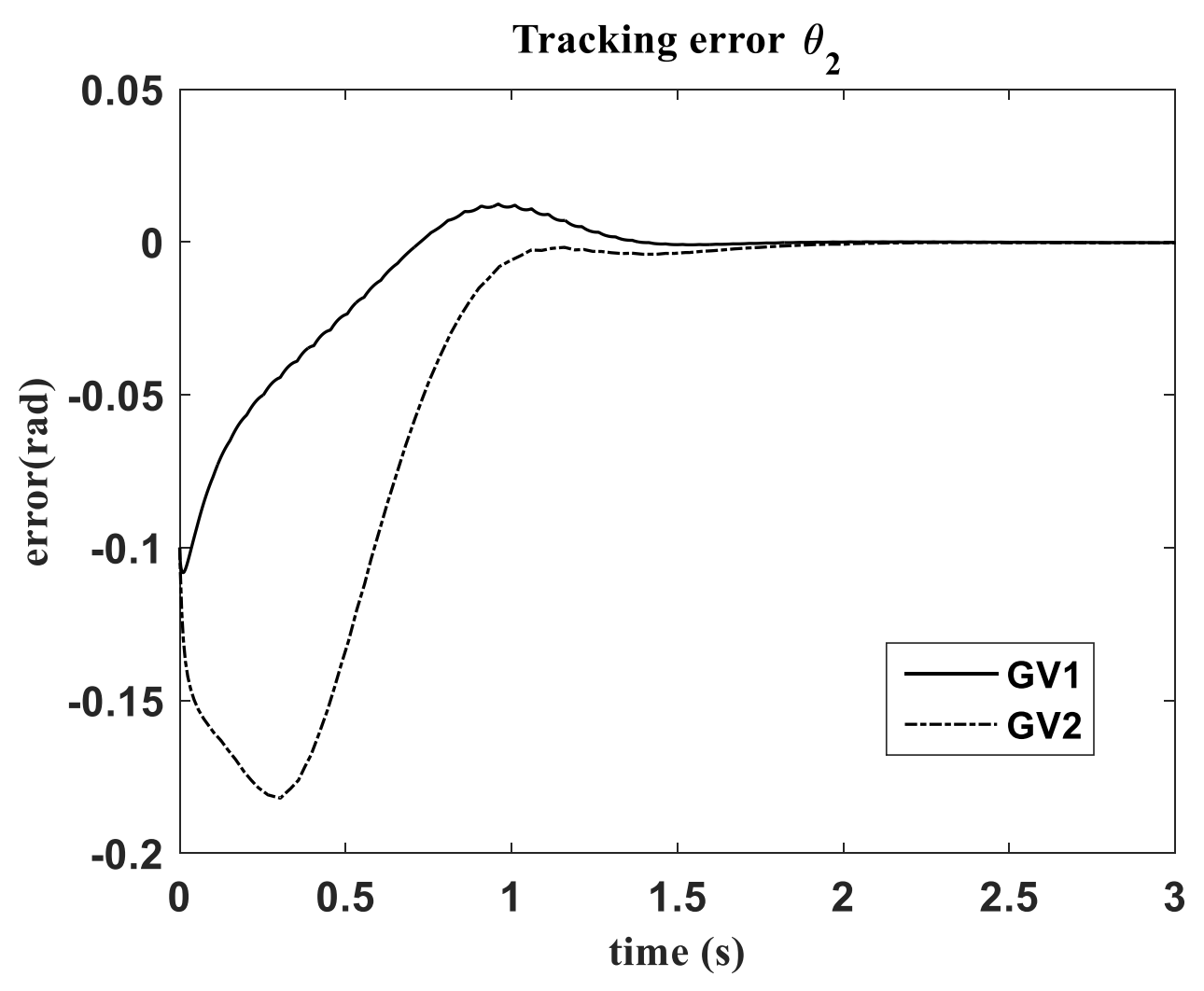

Figure 8: tracking error of $\theta_{2}$, stability prove 


\section{Simulation}

The properties of a human leg are used for the simulations [19]. Table in Fig 6. present the values of the properties of the thigh and the leg used in this study (male weight of $80.5 \mathrm{~kg}$ and mean stature of $1.77 \mathrm{~m}$;). CG inertia is the moment of inertia around the center of gravity, CG distance is measured from the proximal point, representing the distances of $l_{C G 1}$ and $l_{C G 2}$ from figure 3 . The center of mass and the proximal and distal endpoints are assumed to be aligned.

\begin{tabular}{|l|l|r|}
\hline LIMB & Property & Value \\
\hline \multirow{5}{*}{ THIGH (limb1) } & Mass $(\mathrm{kg})$ & 9.902 \\
\cline { 2 - 3 } & Length(m) & 0.432 \\
\cline { 2 - 3 } & CG Inertia $\left(\mathrm{kg}^{*} \mathrm{~m}^{2}\right)$ & 0.166 \\
\cline { 2 - 3 } & CG distance (m) & 0.185 \\
\hline \multirow{5}{*}{ LEG (limb 2) } & Mass $(\mathrm{kg})$ & 3.864 \\
\cline { 2 - 3 } & Length(m) & 0.433 \\
\cline { 2 - 3 } & CG Inertia (kg*m²) & 0.057 \\
\cline { 2 - 3 } & CG distance (m) & 0.178 \\
\hline
\end{tabular}

Figure 9: Table of Body Segment Inertial Properties (BSIP's)

Three conditions of cadence (gait cycle duration[17]) are evaluated in simulation (Fig. 10). Values for normal walk cadence is taken form [16] and slow and very slow walk cadences are taken from [20].

\begin{tabular}{|l|r|r|r|}
\hline Walk & $\begin{array}{l}\text { Cadence } \\
\text { (steps/min) }\end{array}$ & $\begin{array}{l}\text { Stride } \\
\text { time (s) }\end{array}$ & $\begin{array}{l}\text { Swing phase } \\
\text { time (s) }\end{array}$ \\
\hline $\begin{array}{l}\text { Very Slow } \\
\text { (VS) }\end{array}$ & 63 & 1.9 & 0.8 \\
\hline Slow (S) & 80 & 1.5 & 0.6 \\
\hline Normal (N) & 110 & 1.1 & 0.4 \\
\hline
\end{tabular}

Figure 10: Cadence table

The simulation was implemented in Simulink and Simmechanics (fig. 11). The control blocks call Matlab functions with equations of the inverse dynamics as well as the position and velocity gain matrices. The system is simulated using Simmechanics. The inertial properties 
and friction constants $\left(1\left[\frac{\mathrm{kg} \cdot \mathrm{m}^{2}}{\mathrm{~s} \cdot \mathrm{rad}}\right]\right)$ are identical for the control blocks and for the Simmechanics model.

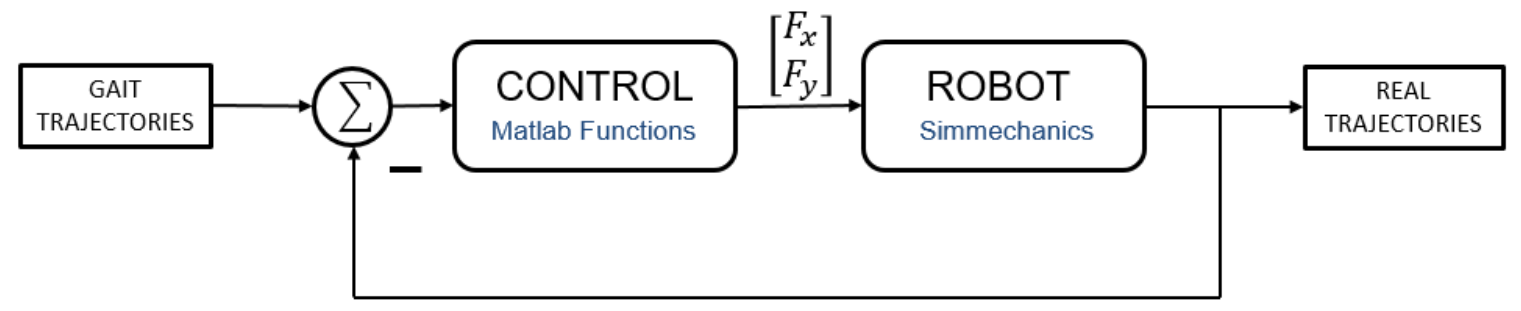

Figure 11: Simulation Model

Gain matrices $K_{p}$ and $K_{v}$ are diagonal. The values used for different simulation cases are presented in Fig. 12. Gait results are the trajectory tracking of the swing phase of the gait graphs Fig. 2 and Fig. 3. A comparison between different gain values is made according to Fig. 12. The GV1 is the pre-compensated case, without any feedback, because the values of the gains are zero for this case. GV2 has more derivative gain value and GV3 is with more proportional value.

\begin{tabular}{|l|l|l|l|l|}
\hline \multirow{2}{*}{$\begin{array}{l}\text { Gain } \\
\text { Values }\end{array}$} & \multicolumn{2}{|c|}{$\mathrm{K}_{\mathrm{p}}$} & \multicolumn{2}{c|}{$\mathrm{K}_{\mathrm{v}}$} \\
\cline { 2 - 5 } $\mathrm{K}_{\mathrm{p} 1}$ & $\mathrm{~K}_{\mathrm{p} 2}$ & $\mathrm{~K}_{\mathrm{v} 1}$ & $\mathrm{~K}_{\mathrm{v} 2}$ \\
\hline $\mathrm{GV}_{1}$ & 0 & 0 & 0 & 0 \\
\hline $\mathrm{GV}_{2}$ & 100 & 100 & 500 & 500 \\
\hline $\mathrm{GV}_{3}$ & 785 & 785 & 250 & 250 \\
\hline \multicolumn{6}{|c}{ Figure 12: Gain Values table }
\end{tabular}

\section{Results}

In this section the results from the simulation in Simulink are presented. Two types of simulation are performed, using the gait trajectories and using trajectories proposed in [18] in order to compare with other controller.

\section{Gait Results}

\section{Trajectory Tracking}

Fig. 13 and Fig. 14 show the graph for the trajectory tracking of the gait. Simulations are performed at a normal cadence Fig. 7 and only the swing phase is simulated. 


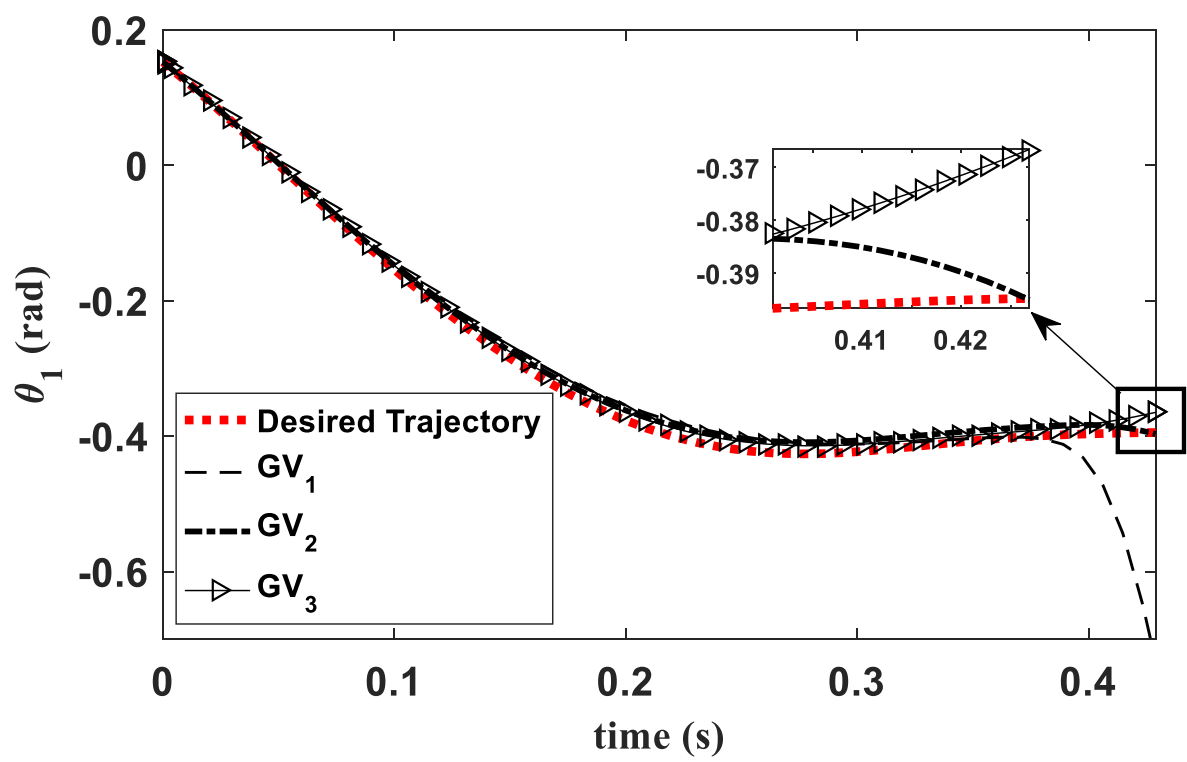

Figure 13: Trajectory tracking for $\theta_{1}$

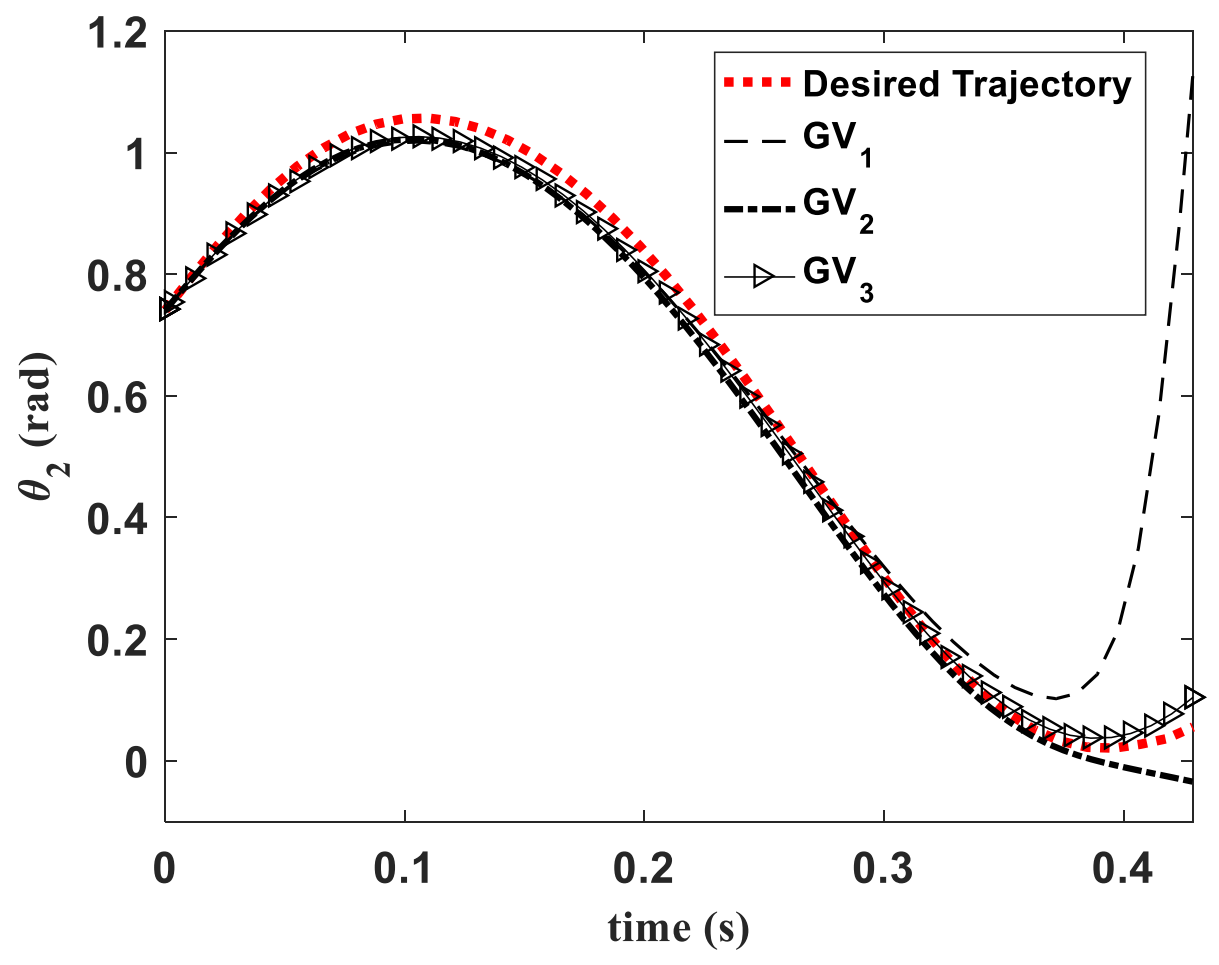

Figure 14: Trajectory tracking for $\theta_{2}$

\section{Tracking Error}

Fig 15. And Fig. 16 show the tracking error of the trajectory tracking. Error defines as the difference between the desired trajectory and the real one. 


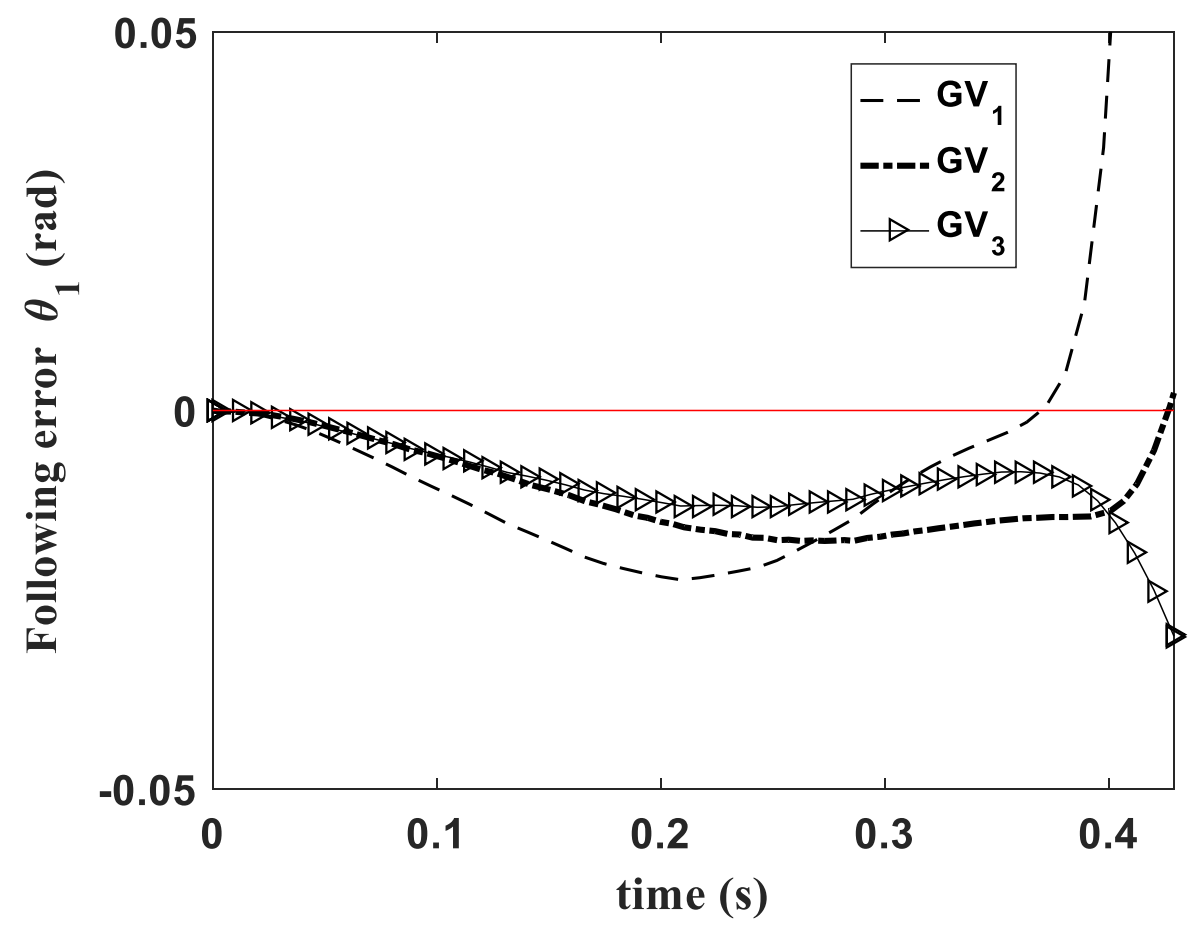

Figure 15: Tracking error of $\theta_{1}$

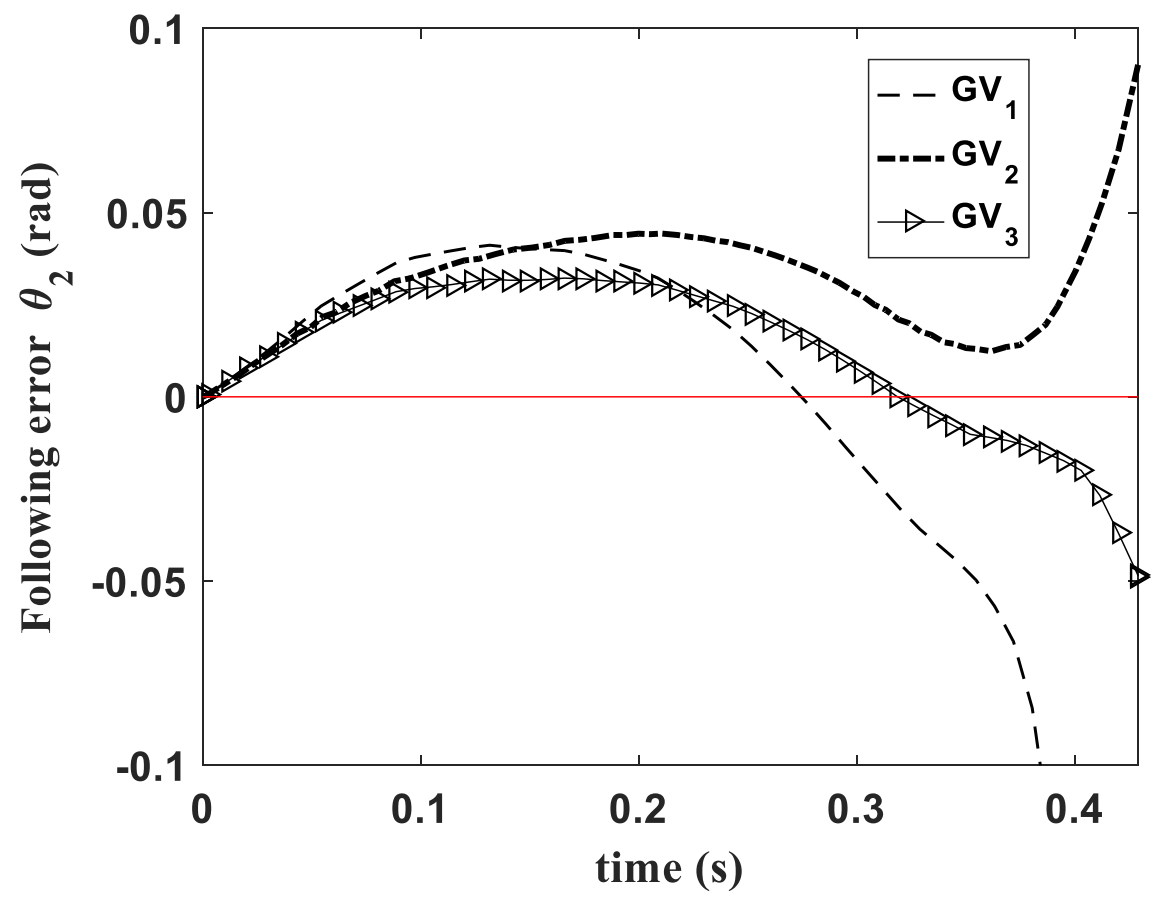

Figure 16: Tracking error of $\theta_{1}$

\section{Motion of the cart}

The $\mathrm{x}$ and $\mathrm{y}$ variables are not controllable variables, that means that the model as planned does not allow us to manage what happen with those variables. And due to the application of 
this model (rehabilitation) the work space is an important aspect. Fig. 17 presents the behavior of the cart depending on the cadence (Fig. 10) of the trajectory that it is being followed. Red dot indicates where the motion begins, time of the motion varies depending on the cadence.

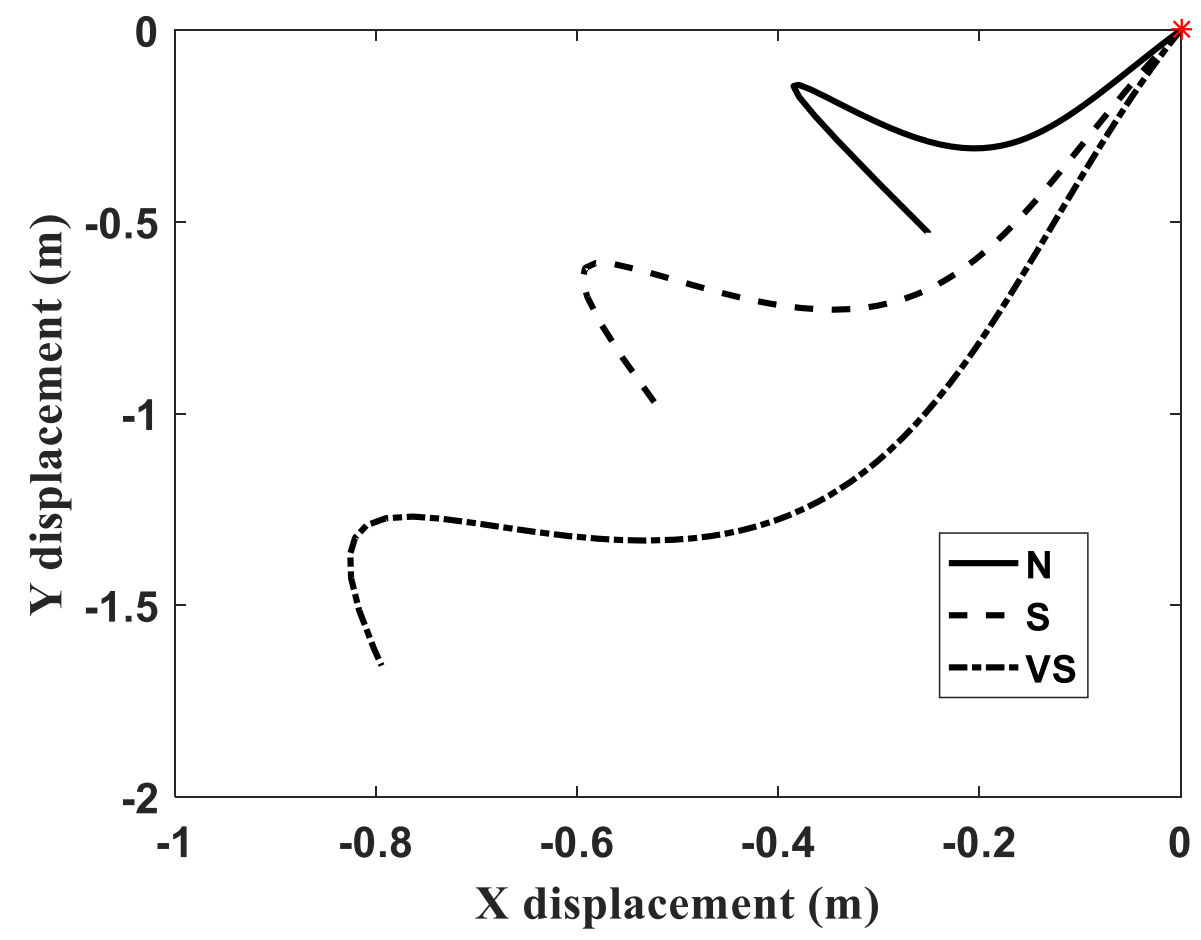

Figure 17: Motion of the cart

\section{Design and construction of a human leg}

To make the simulations and the future experimental validation, the limbs of the pendulum were design with the parameters and inertial properties of a real human leg. The paper "Adjustments to McConville et al. and Young et al. body" [19] provides the body segment inertial properties (BSIP's).

The first parameters are the center of mass (CM) and the dimensions of the limbs. Also, we must stablish the coordinate system used. Fig. 18 shows the locations of selected anatomical landmarks and the orientations of the segment coordinate system (SCSs) built from these landmarks. 


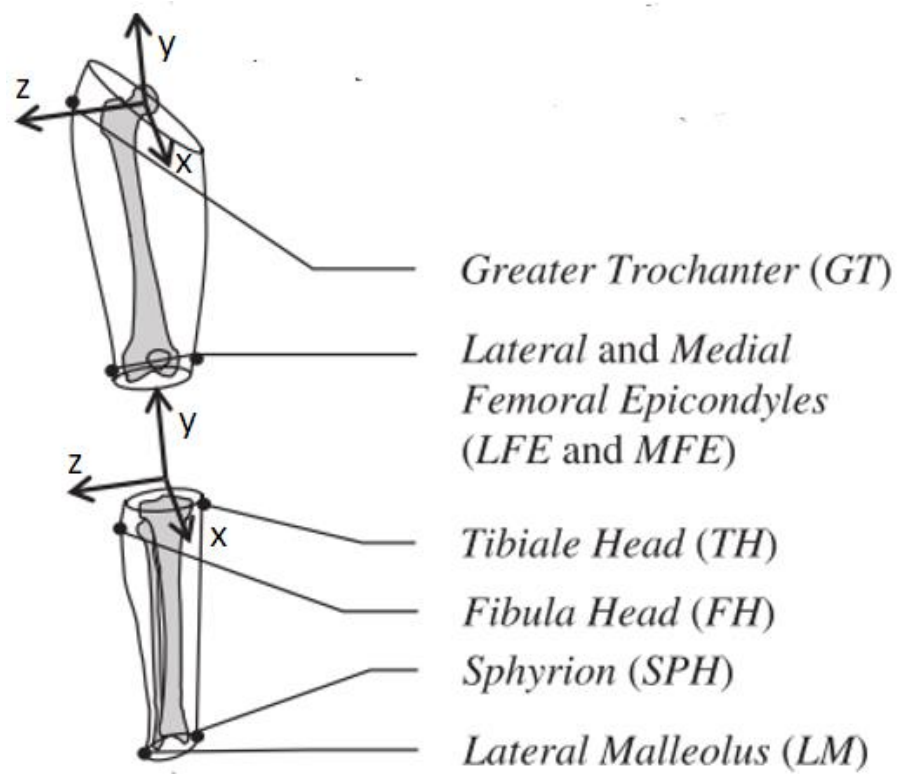

Figure 18: Locations of selected anatomical landmarks and SCSs

The segment coordinate system (SCS) of each limb is shown in figure 19. Fig. 20 presents the location of the points represented in Fig. 19 with the SCS as reference. CM is also presented.

\begin{tabular}{|c|c|c|c|c|c|c|c|}
\hline \multirow[b]{2}{*}{ Segment } & \multirow[b]{2}{*}{ Anatomical landmark } & \multicolumn{3}{|l|}{ Female } & \multicolumn{3}{|l|}{ Male } \\
\hline & & $X$ (in $\mathrm{mm})$ & $Y$ (in $\mathrm{mm})$ & $Z$ (in $\mathrm{mm}$ ) & $X$ (in $\mathrm{mm})$ & $Y($ in $\mathrm{mm})$ & $Z$ (in $\mathrm{mm}$ ) \\
\hline \multirow[t]{4}{*}{ Thigh } & Greater Trochanter (GT) & -19 & 8 & 87 & -40 & 6 & 101 \\
\hline & Lateral Femoral Epicondyle (LFE) & 0 & -375 & 57 & 0 & -431 & 57 \\
\hline & Medial Femoral Epicondyle (MFE) & 0 & -382 & -57 & 0 & -432 & -57 \\
\hline & Centre Of Mass (COM) & -29 & -143 & 3 & -18 & -185 & 14 \\
\hline \multirow[t]{5}{*}{ Leg } & Tibiale Head (TH) & 8 & -22 & -39 & 21 & -27 & -42 \\
\hline & Fibula Head (FH) & 0 & -40 & 60 & 0 & -23 & 47 \\
\hline & Sphyrion $(S P H)$ & 10 & -387 & -31 & 21 & -434 & -33 \\
\hline & Lateral Malleolus (LM) & -10 & -390 & 31 & -21 & -433 & 33 \\
\hline & Centre Of Mass (COM) & -19 & -157 & 12 & -21 & -178 & 3 \\
\hline
\end{tabular}

Figure 19: Body segment dimensions

This work presents the properties as a scaling factor, to find the values depending on the mass of the person. So, we assume that the person is a male of $80,5 \mathrm{~kg}$ (mean of the study performed in this paper). Fig. 21 presents the properties as a scaling factor. 


\begin{tabular}{|l|r|l|r|}
\hline \multicolumn{2}{|c|}{$\begin{array}{l}\text { THIGH (Scaling } \\
\text { Factor\%) }\end{array}$} & \multicolumn{2}{c|}{ LEG(Scaling Factor\%) } \\
\hline rxx & 29 & rxx & 28 \\
\hline ryy & 15 & ryy & 10 \\
\hline rzz & 30 & rzz & 28 \\
\hline rxy & 7 & rxy & 4 \\
\hline rxz & -2 & rxz & 2 \\
\hline ryz & -7 & ryz & 5 \\
\hline mass & 12,3 & mass & 4,8 \\
\hline \multicolumn{2}{|c|}{ Figure 20: Inertial properties as a Scaling Factor } \\
\cline { 2 - 3 }
\end{tabular}

The mass limb scaling factor works as a percentage of the total mass of the person, but to find the inertia matrix an equation needs to be applied.

$$
r_{i j}=\left(\frac{1}{L}\right) \sqrt{\frac{I_{i j}}{m}}
$$

Where $\mathrm{L}$ is the segment length or limb length and $\mathrm{m}$ is the segment mass or limb mass. So, to find each value of the inertia matrix

$$
I_{i j}=\left(r_{i j} L\right)^{2} m
$$

Now, the matrices of the inertia respect to the center of mass are presented in $\left[\mathrm{kg} \cdot \mathrm{m}^{2}\right]$

$$
\begin{aligned}
I_{\text {THIGH }} & =\left[\begin{array}{ccc}
0,155 & 0,009 & 0,0007 \\
0,009 & 0,042 & -0,009 \\
0,0007 & -0,009 & 0,166
\end{array}\right] \\
I_{L E G} & =\left[\begin{array}{ccc}
0,057 & 0,001 & 0,0003 \\
0,001 & 0,007 & 0,002 \\
0,0003 & 0,002 & 0,057
\end{array}\right]
\end{aligned}
$$

To simplify the design, and because the model of the pendulum is in 2 dimensions, only the moment of inertia around the z-axis is needed. So, to resume the previous work and to present only the needed information to the design Fig. 21 has the important properties.

\begin{tabular}{|l|r|r|}
\hline Property & \multicolumn{1}{|l|}{ THIGH } & LEG \\
\hline mass $(\mathrm{kg})$ & 9,90 & 3,86 \\
\hline Length $(\mathrm{mm})$ & 432 & 433 \\
\hline Inertia CM $\left(\mathrm{kg}^{*} \mathrm{~m}^{2}\right)$ & 0,17 & 0,057 \\
\hline CM distance $(\mathrm{mm})$ & 185 & 178 \\
\hline \multicolumn{2}{|c|}{ Figure 21: properties of the double-pendulum } \\
\hline
\end{tabular}

Using Autodesk Inventor, two limbs (thigh and leg) are design to guarantee the properties shown in Fig. 21. Fig. 22 and Fig. 23 present the results of the design, blueprints are presented in annexes. 


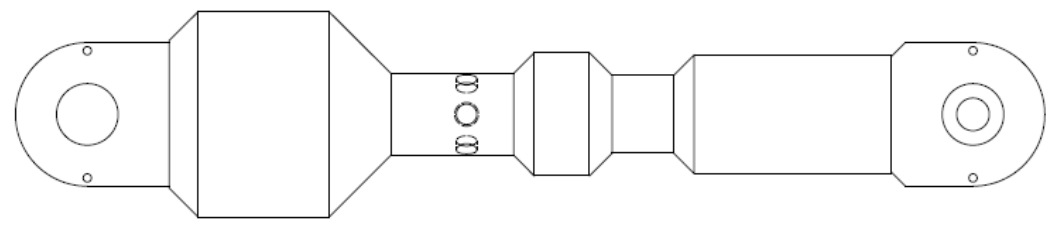

Figure 22: Thigh design

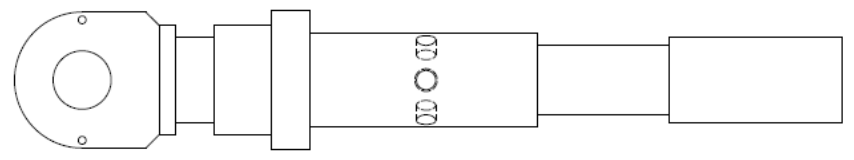

Figure 23: Leg design

The design was thought to be assemble with bearings and to have the option of changing the properties with threaded holes in the center of mass. Fig. 24 is a photo of the assembly of the leg with the Stewart platform.

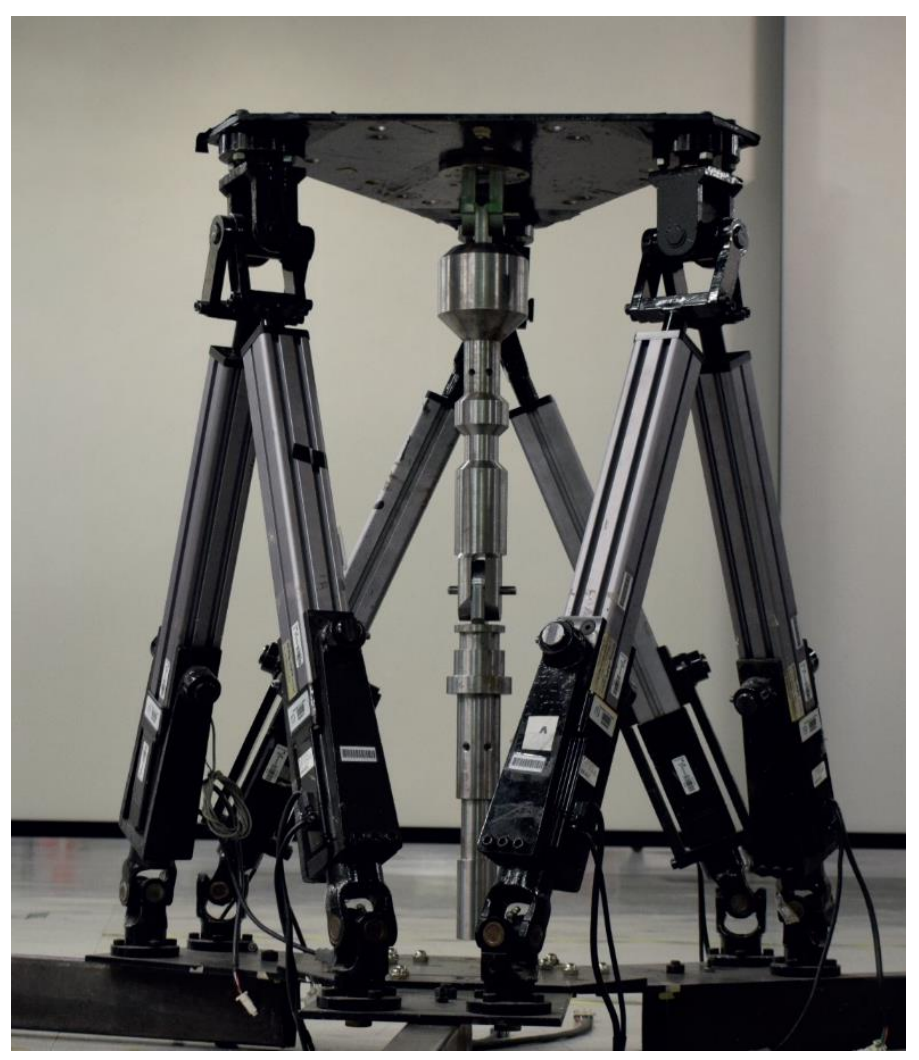

Figure 24: Assembly of the leg and the Stewart platform 


\section{Analysis}

\section{Stability Results}

At the beginning of this document a mathematical prove was presented, where we found the conditions that guarantee the asymptotic stability. On the section of Stability Results a tuning was made for the trajectories presented in Fig. 6. The results of the tracking error of that tuning are presented in Fig. 7 and Fig. 8, as well as a different set of values taken from[19]

The GV1 reaches the stability in the same time as the GV2, but for $\theta_{2}$ the behavior is not desirable. For $\theta_{1}$ the behavior is similar in both cases.

For these simulations an error in the initial conditions is imposed and the behavior in time is studied. The set of values GV1 show a better behavior and it reaches the trajectory faster and with better behavior in the case of $\theta_{2}$. It takes around 1.5 seconds to reach stability.

\section{Trajectory Tracking}

Figure 13 to 16 present the results of the trajectory tracking control. Three gain configurations are tested.

The first configuration is the open-loop controller, with only the pre-compensation from the dynamics. This controller is adequate for values of $\theta_{2}$ far from zero, because the dynamic equations present a singularity in that point. Following error graphs show this problem more clearly. The error is low at the beginning of the trajectory, but increases at the end when $\theta_{2}$ is very close to zero.

With the addition of a PD in the second and third cases, the problem is solved. $\mathrm{GV}_{2}$ works better on following the trajectory of $\theta_{1}$, while $\mathrm{GV}_{3}$ follows better the trajectory of $\theta_{2}$. In general, $\mathrm{GV}_{3}$ produce smaller following error. Bigger gain values do not generate important improvement in error reduction but result in bigger applied forces.

Fig. 15 and Fig. 16 seem to have a non-desireable behavior, but as shown in the simulations

of stability, it takes around 1.5 seconds to reach stability. So, it is expected that in the 0.4 seconds of the gait simulation does not reach an error equal to zero in time. Even though, the errors of the tracking error do not overcome the 5\% respect to the amplitude of the trajectory.

\section{Motion of the cart}


As seen on figure 17 the motion of the cart, can be too wide to be generated for a robot, especially if it will be promoted on a human hip. The only acceptable case is the normal walk cadence, which covers an area of $0.5 \times 0.4$ meters. This motion has to be limited since the hip will be pushed only during the swing phase, and the patient will complete the stance phase over a treadmill. This motion is also affected by the friction in the joints, but for this study only the gait cadence is taken into account.

\section{Conclusion}

This study presents the trajectory tracking control for a double pendulum. The dynamical system was found with the Lagrange formulation. Simulations show good results for tracking gait trajectories for a PD with pre-compensation controller. Motion of the cart was also acceptable for a normal walk cadence. The controller was proven stable, mathematically and with simulations. The limbs of the double-pendulum were built with the characteristics of a real human leg, those characteristics were used in the simulations.

Future work will be to validate the controller using a Stewart platform and an instrumented double pendulum. (Fig 24).

The use of this controller for a rehabilitation robot must include an additional study on the interaction forces between the robot and the patient. This aspect can be even more relevant than reducing the following error of the trajectory tracking. 


\section{References}

[1] A. Dabiri, S. Sabet, M. Poursina, D. G. Armstrong, and P. E. Nikravesh, "An optimal Stewart platform for lower extremity robotic rehabilitation," in Proceedings of the American Control Conference, 2017.

[2] T. Onodera, M. Ding, H. Takemura, and H. Mizoguchi, "Design and development of Stewart platform-type assist device for ankle-foot rehabilitation," in Proceedings of the 20121 st International Conference on Innovative Engineering Systems, ICIES 2012, 2012.

[3] V. J. F. JF, R. Kruidhof, E. E. G. Hekman, R. Ekkelenkamp, and E. H. F. Van Asseldonk, "Design and evaluation of the LOPES exoskeleton robot for interactive gait rehabilitation.," IEEE Trans. Neural Syst. Rehabil. Eng., vol. 15, no. 3, pp. 379-386.

[4] A. S. M. M, A. M. N. Rashty, C. Rode, and A. Seyfarth, "Reconstruction of human swing leg motion with passive biarticular muscle models.," Hum. Mov. Sci., vol. 52, pp. 96-107.

[5] B.-L. Yousef, G. A, E. M, N. M, and S. A, "Dynamics and Control of Locomotion of One Leg Walking as Self-Impact Double Pendulum.” 2011.

[6] E. K. K and I. Nilkhamhang, "Swing Phase Control of Semi-Active Prosthetic Knee Using Neural Network Predictive Control With Particle Swarm Optimization.," IEEE Trans. Neural Syst. Rehabil. Eng., vol. 24, no. 11, pp. 1169-1178, 2016.

[7] N. YS, M. A. VN, and W. A. G. 5th, "The Effects of the Inertial Properties of Above-Knee Prostheses on Optimal Stiffness, Damping, and Engagement Parameters of Passive Prosthetic Knees. TT -," J. Biomech. Eng. TA -, vol. 138, no. 12, 2016.

[8] S. Alexander, J. Austin, and H. Geyer, Evaluation of Decentralized Reactive SwingLegControl on a Powered RoboticLeg. 2015.

[9] W. Jia-Jun, "Simulation studies of inverted pendulum based on PID controllers," Simul. Model. Pract. Theory, vol. 19, no. 1, pp. 440-449, 2011.

[10] Y. Bai, Z. Chen, and X. Ruan, "Complex Trajectory Tracking of Inverted Pendulum Using PID and LQR." International Conference on Manufacturing Science and Information Engineering, Beijing, 2017.

[11] C. He, Y. Fang, and N. Sun, "A swing constrained time-optimal trajectory planning strategy for double pendulum crane systems," NONLINEAR Dyn., vol. 89, no. 2, pp. 1513-1524, 2017.

[12] N. Sun, Y. Wu, Y. Fang, and H. Chen, "Nonlinear Antiswing Control for Crane Systems With Double-Pendulum Swing Effects and Uncertain Parameters: Design and Experiments," IEEE Trans. Autom. Sci. Eng., vol. 15, no. 3, pp. 1413-1422, 2018.

[13] M. Kumar, B. Singh, S. Member, and B. P. Singh, "Pre-compensated Controller Motor," pp. 2-6.

[14] B.-L. Yousef, M. Eghtesad, A. Khoogar, and A. Mohammad-Zadeh, "Tracking Control of A Human Swing Leg Considering Self-Impact Joint Constraint by Feedback Linearization Method," Control Eng. Appl. INFORMATICS, vol. 17, no. 1, pp. 99-110, 2015.

[15] Xinjilefu, V. Hayward, and H. Michalska, Hybrid Stabilizing Control for the Spatial Double Inverted Pendulum. BERLIN: SPRINGER-VERLAG BERLIN, 2010. 
[16] G. Bovi, M. Rabuffetti, P. Mazzoleni, and M. Ferrarin, “A multiple-task gait analysis approach: Kinematic, kinetic and EMG reference data for healthy young and adult subjects TT -," Gait Posture TA -, vol. 33, no. 1, pp. 6-13, 2011.

[17] R. T. A.-T. T.- Baker, "Measuring walking : a handbook of clinical gait analysis." Mac Keith Press, London :, 2013.

[18] R. Kelly and V. Santibáñez, Control de Movimiento de Robots Manipuladores. Madrid: Pearson Education, 2003.

[19] R. Dumas, L. Chèze, and J. P. Verriest, "Adjustments to McConville et al. and Young et al. body segment inertial parameters," Journal of Biomechanics, vol. 40, no. 3. pp. 543-553, 2007.

[20] L. R. Nascimento, C. Q. de Oliveira, L. Ada, S. M. Michaelsen, and L. F. Teixeira-Salmela, "Walking training with cueing of cadence improves walking speed and stride length after stroke more than walking training alone: a systematic review TT -," journal of

physiotherapy, vol. 61. pp. 10-15, 2015. 


\section{Annexes}

In this section, annexes of the project are presented.

\section{Dynamical model matrices}

$$
\begin{gathered}
M=\left[\begin{array}{ll}
M_{11} & M_{12} \\
M_{21} & M_{22}
\end{array}\right] \quad C=\left[\begin{array}{ll}
C_{11} & C_{12} \\
C_{21} & C_{22}
\end{array}\right] \quad \boldsymbol{g}=\left[\begin{array}{l}
g_{1} \\
g_{2}
\end{array}\right] \\
p=\frac{\left(M+m_{1}+m_{2}\right)}{m_{2} l_{2 G} s_{2}\left(m_{2} l_{1}+m_{1} l_{1 G}\right)}
\end{gathered}
$$

$M_{11}=-p\left(-l_{1}^{2} l_{2 G} m_{2}^{2} s_{12}-I_{1} l_{2 G} m_{2} s_{12}+l_{1} l_{2 G}^{2} m_{2}^{2} s_{1}+I_{2} l_{1} m_{2} s_{1}+I_{2} l_{1 G} m_{1} s_{1}-l_{1} l_{2 G}^{2} m_{2}^{2} s_{12} c_{2}+\right.$ $\left.l_{1}^{2} l_{2 G} m_{2}^{2} c_{2} s_{1}-l_{1 G}^{2} l_{2 G} m_{1} m_{2} s_{12} l_{1 G} l_{2 G}^{2} m_{1} m_{2} s_{1}+l_{1} l_{1 G} l_{2 G} m_{1} m_{2} c_{2} s_{1}\right)-m_{1} l_{1 G} c_{1}-m_{2} l_{1} c_{1}-$ $l_{2 G} c_{12} m_{2}$

$M_{12}=-p\left(l_{1} l_{2 G}^{2} m_{2}^{2} s_{1}+I_{2} l_{1} m_{2} s_{1}+I_{2} l_{1 G} m_{1} s_{1}-l_{1} l_{2 G}^{2} m_{2}^{2} s_{12} c_{2}+l_{1 G} l_{2 G}^{2} m_{1} m_{2} s_{1}\right)-m_{2} l_{2 G} c_{12}$

$M_{21}=\frac{p}{2}\left(-l_{1}^{2} l_{2 G} m_{2}^{2} c_{12}-2 I_{1} l_{2 G} m_{2} c_{12}+l_{1} l_{2 G}^{2} m_{2}^{2} c_{1}+2 I_{2} l_{1} m_{2} c_{1}+2 I_{2} l_{1 G} m_{1} c_{1}+\right.$

$l_{1}^{2} l_{2 G} m_{2}^{2} \cos \left(\theta_{1}-\theta_{2}\right)-l_{1} l_{2 G}^{2} m_{2}^{2} \cos \left(\theta_{1}+2 \theta_{2}\right)-2 l_{1 G}^{2} l_{2 G} m_{1} m_{2} c_{12}+2 l_{1 G} l_{2 G}^{2} m_{1} m_{2} c_{12}+$

$\left.l_{1} l_{1 G} l_{2 G} m_{1} m_{2} \cos \left(\theta_{1}-\theta_{2}\right)\right)-m_{2}\left(l_{1} s_{1}+l_{2 G} s_{12}\right)-m_{1} l_{1 G} s_{1}$

$M_{22}=\frac{p}{2}\left(l_{1} l_{2 G}^{2} m_{2}^{2} c_{1}+2 I_{2} l_{1} m_{2} c_{1}+2 I_{2} l_{1 G} m_{1} c_{1}-l_{1} l_{2 G}^{2} m_{2}^{2} \cos \left(\theta_{1}+2 \theta_{2}\right)+2 l_{1 G} l_{2 G}^{2} m_{1} m_{2} c_{1}\right)-$ $m_{2} l_{2 G} s_{12}$

$C_{11}=-p\left(-d_{1} l_{2 G} m_{2} s_{12}+l_{1}^{2} l_{2 G} m_{2}^{2} \dot{\theta}_{1} s_{1} s_{2}+l_{1} l_{2 G}^{2} m_{2}^{2} \dot{\theta}_{1} s_{12} s_{2}+2 l_{1} l_{2 G}^{2} m_{2}^{2} \dot{\theta}_{2} s_{12} s_{2}+\right.$ $\left.l_{1} l_{1 G} l_{2 G} m_{1} m_{2} \dot{\theta}_{1} s_{1} s_{2}\right)+m_{1} l_{1 G} \dot{\theta}_{1} s_{1}+m_{2}\left(\dot{\theta}_{1}+2 \dot{\theta}_{2}\right) l_{2 G} s_{12}+m_{2} l_{1} \dot{\theta}_{1} s_{1}$

$C_{12}=-p\left(d_{2} l_{2 G} m_{2} s_{12}+d_{2} l_{1} m_{2} s_{1}+d_{2} l_{1 G} m_{1} s_{1}+l_{1} l_{2 G}^{2} m_{2}^{2} \dot{\theta}_{2} s_{12} s_{2}\right)+m_{2} l_{2 G} s_{12} \dot{\theta}_{2}$

$C_{21}=\frac{p}{2}\left(l_{1} l_{2 G}^{2} m_{2}^{2} \dot{\theta}_{1} \sin \left(\theta_{1}+2 \theta_{2}\right)-l_{1}^{2} l_{2 G} m_{2}^{2} \dot{\theta}_{1} \sin \left(\theta_{1}-\theta_{2}\right)-l_{1} l_{2 G}^{2} m_{2}^{2} \dot{\theta}_{1} s_{1}-2 d_{1} l_{2 G} m_{2} c_{12}+\right.$ $l_{1}^{2} l_{2 G} m_{2}^{2} \dot{\theta}_{1} s_{12}-2 l_{1} l_{2 G}^{2} m_{2}^{2} \dot{\theta}_{2} s_{1}+2 l_{1} l_{2 G}^{2} m_{2}^{2} \dot{\theta}_{2} \sin \left(\theta_{1}+2 \theta_{2}\right)+l_{1} l_{1 G} l_{2 G} m_{1} m_{2} \dot{\theta}_{1} s_{12}-$ $\left.l_{1} l_{1 G} l_{2 G} m_{1} m_{2} \dot{\theta}_{1} \sin \left(\theta_{1}-\theta_{2}\right)\right)-m_{2} l_{2 G} c_{12}\left(\dot{\theta}_{1}+2 \dot{\theta}_{2}\right)-m_{2} l_{1} \dot{\theta}_{1} c_{1}-m_{1} l_{1 G} \dot{\theta}_{1} c_{1}$

$C_{22}=\frac{p}{2}\left(-l_{1} l_{2 G}^{2} m_{2}^{2} \dot{\theta}_{2} s_{1}+l_{1} l_{2 G}^{2} m_{2}^{2} \dot{\theta}_{2} \sin \left(\theta_{1}+2 \theta_{2}\right)+2 d_{2} l_{2 G} m_{2} c_{12}+2 d_{2} l_{1} m_{2} c_{1}+\right.$ $\left.2 d_{2} l_{1 G} m_{1} c_{1}\right)-m_{2} l_{2 G} c_{1} \dot{\theta}_{2}$

$$
\begin{gathered}
g_{1}=0 \\
g_{2}=\frac{p}{2}\left(2 g l_{1} l_{2 G} m_{2}^{2} s_{2}+2 g l_{1 G} l_{2 G} m_{1} m_{2} s_{2}\right)-\left(M+m_{1}+m_{2}\right) g
\end{gathered}
$$

\section{Blueprints}




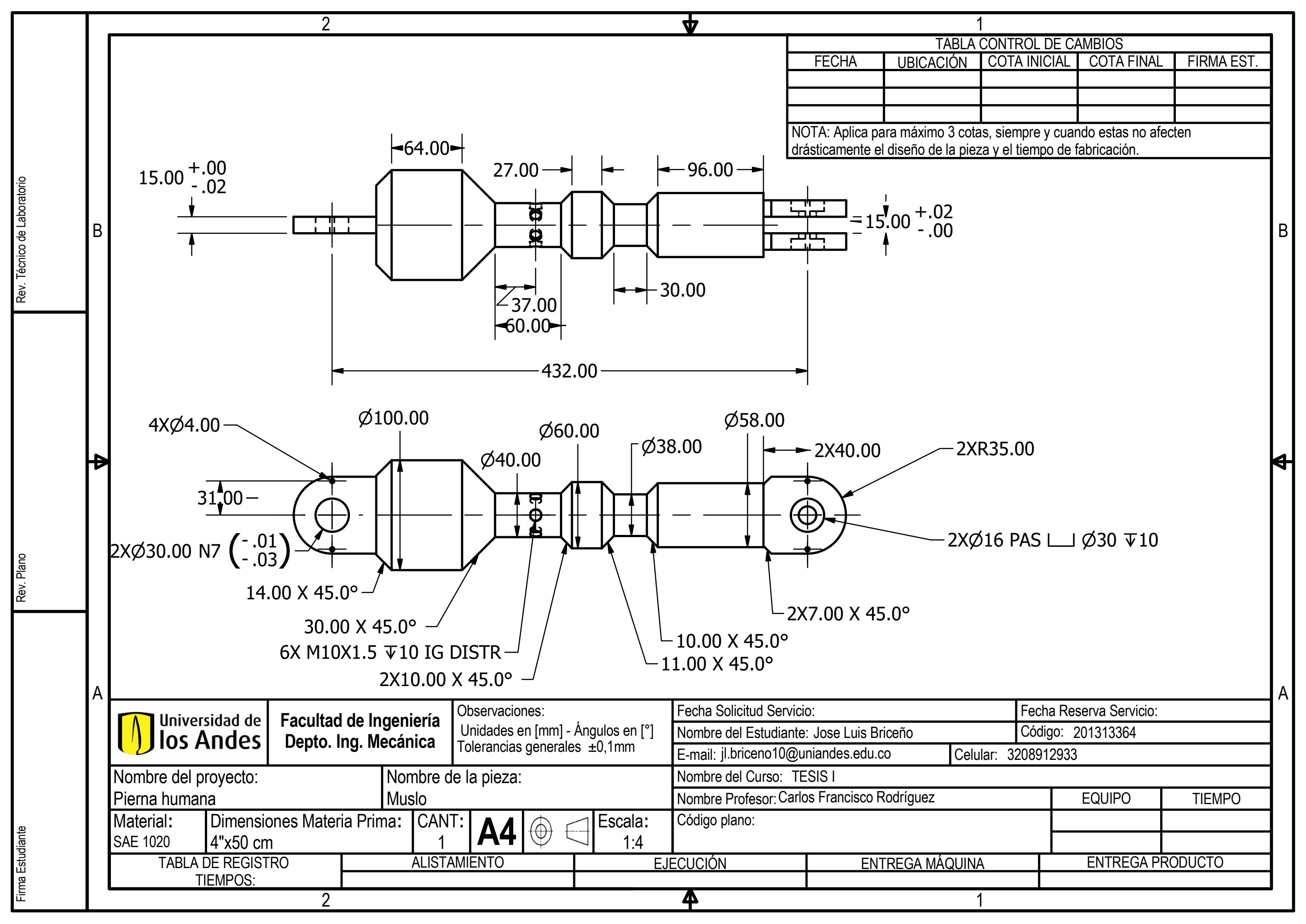




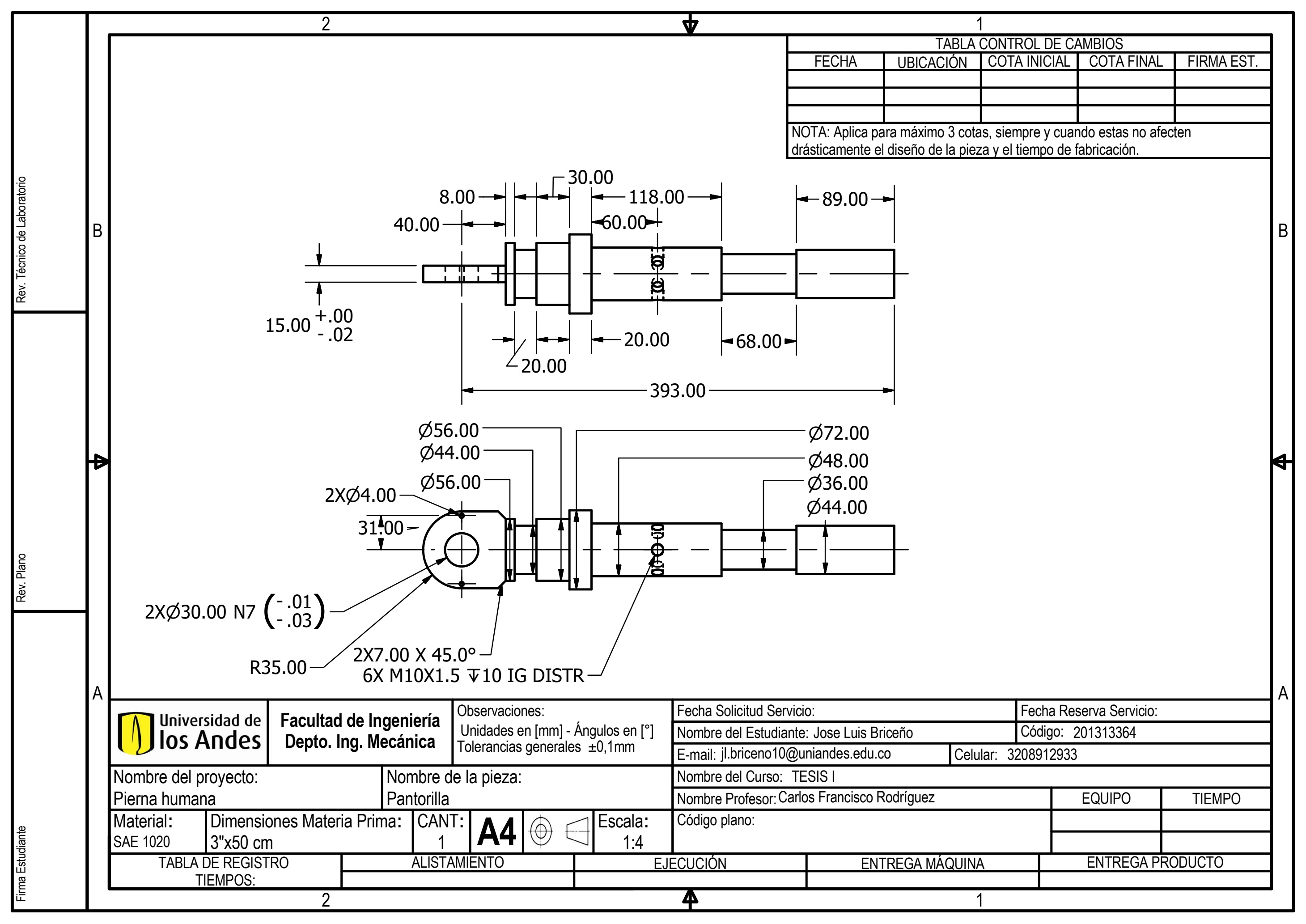

